\title{
Furanchalcone-biphenyl hybrids: synthesis, in silico studies, antitrypanosomal and cytotoxic activities
}

Elisa García ${ }^{1}$, Rodrigo Ochoa ${ }^{2}$, Laura Conesa-Milián ${ }^{2}$, Miguel Carda ${ }^{2}$, Andrés Yepes ${ }^{1}$, Isabel

Vásquez ${ }^{1}$, Iván D. Vélez ${ }^{3}$, Sara M. Robledo ${ }^{3, *}$, Wilson Cardona-G ${ }^{1, *}$

1. Chemistry of Colombian Plants, Institute of Chemistry, Exact and Natural Sciences School,

Universidad de Antioquia-UdeA, Calle 70 No. 52-21, A.A 1226, Medellín, Colombia

2. PECET-Medical Research Institute, School of Medicine, Universidad de Antioquia-UdeA. Calle 70

No. 52-21, A.A 1226 Medellín, Colombia

3. Department of Inorganic and Organic Chemistry, Jaume I University, E-12071 Castellón, Spain

*Author to whom correspondence should be addressed; e-mail: sara.robledo@udea.edu.co; wilson.cardona1@udea.edu.co; phone: +574-2196503 (S.M.R), +574-2195653 (W.C); Fax: +5742196511 (S.M.R), +57-42330120 (W.C) 


\begin{abstract}
The synthesis, anti-trypanosomal and cytotoxic activities of seventeen furanchalcone derivatives are described herein. The structure of the synthesized products was elucidated by a combination of spectrometric analyses. The synthesized compounds were evaluated against Trypanosoma cruzi, which is the pathogenic species to humans. Cytotoxicity was evaluated against human U-937 macrophages. Eleven compounds were active against amastigotes of $T$. cruzi with $\mathrm{EC}_{50}$ values lower than $40 \mu \mathrm{M}$. Hybrids 7b-7d and 8a-8g showed better activity than that of benznidazole. Structure Activity Relationship (SAR) showed that the presence of electron withdrawing groups, such as the nitro or fluorine, increased the activity and that the degree of oxygenation is essential for activity. In addition, molecular docking was used to identify a possible protein target for the designed compounds. A spearman correlation of 0.608 between the predicted scores and the experimental data profile the enzyme cruzipain as a potential candidate. Finally, in silico ADMET studies of the arylfuranchalcones showed that these novel compounds have good drug like properties, making them potentially promising agents for antichagasic therapy.
\end{abstract}

Keywords: Chagas disease; trypanosoma cruzi; furanchalcone; hybrids; in silico studies, biphenyl

\title{
Introduction
}


Neglected tropical diseases (NTDs) are diverse group of communicable diseases that prevail in tropical and subtropical conditions in 149 countries. These diseases affect more than one billion people worldwide. NTDs include, among others, Chagas disease (American trypanosomiasis) which is caused by protozoan parasites from Trypanosoma cruzi (T. cruzi) [1].

Chagas disease, also known as American trypanosomiasis, is a potentially life-threatening illness caused by the protozoan parasite Trypanosoma cruzi (T. cruzi). This disease is an important public health problem in Latin America affecting nowadays an estimated 8 million people in 21 countries and spreading by human migration to a number of non-endemic regions. Approximately $20-30 \%$ of the infected population will suffer irreversible cardiovascular, gastrointestinal, and/or neurological problems. The two registered drugs for Chagas disease treatment are Nifurtimox and benznidazole, which require prolonged treatment and have frequent side-effects that can lead to discontinuation of treatment [2]. Based on the above, there is a need to look for new drugs for these diseases in order to provide a shorter treatment course, with fewer side-effects, and also to devise pediatric formulations.

Chalcones are a major class of natural products often found in edible plants. They have received considerable attention due to their wide range of biological actions, probably due to their small structures and Michael acceptor features, which allows them to be tolerant to many biomolecules and at the same time confers on them the necessary reactivity to bind to their biological targets. For this reason, this class of compounds have been used as a scaffold in the development of different pharmacological agents [3-5] including among them those endowed with antitripanosomal activity [6]. Some chalcones depicted in fig. 1 show antiprotozoal activity, such as chalcone 1a, which showed high trypanocidal activity against trypomastigotes of $T$. cruzi and low cytotoxicity (12.2 and 190.9 $\mu \mathrm{M}$, respectively) with a selectivity index of 15.6 [7] and licochalcone A (1b), an oxygenated chalcone isolated from the roots of Glycyrrhiza spp, a Chinese plant, which inhibited the fumarate reductase, a selective target present in the mitochondria of the parasite [8].

Many natural products, such as honokiol (1c) and schisandrin C (1d) (fig. 1), exhibiting a biphenyl moiety in their structures are endowed with many relevant biological activities [9,10]. In this regard, the biphenyl derivative 1e (fig. 1), whose structure is based on that of methylglyoxal bis(guanylhydrazone), was examined for in vitro antitrypanosomal activity and cytotoxicity for human cells. This compound had a $\mathrm{MIC}_{50}$ of $0.14 \mu \mathrm{M}$ for Trypanosoma brucei rhodesiense, and was also active against other trypanosome species, including multidrug-resistant Trypanosoma brucei brucei $\left(\mathrm{MIC}_{50}=11.1 \mu \mathrm{M}\right)[11]$ 

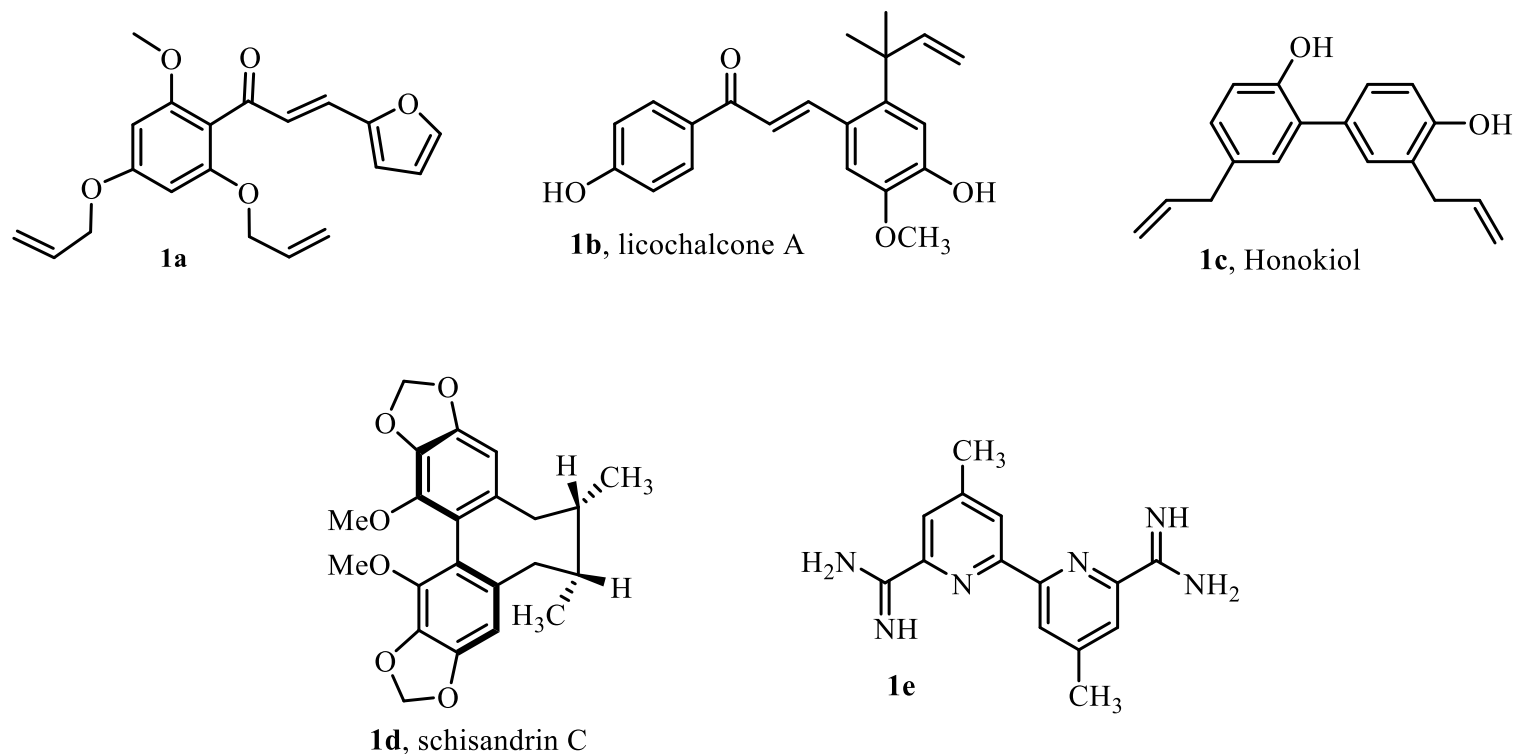

Fig. 1. Chalcones and biphenyls biologically actives

A promising strategy based on hybrid molecules has recently emerged in medicinal chemistry for the discovery and development of new drugs. Hybrid molecules bear in their structures two distinct pharmacophores and can therefore show a dual mode of action $[12,13]$ without necessarily acting on the same biological target [14]. In this sense we have synthesized several triclosan-caffeic acid hybrids and tested them against amastigotes of Trypanosoma cruzi. Among the compounds tested, hybrids $2 \mathrm{a}$ and $\mathbf{2 b}$ exhibited the highest trypanocidal activity $\left(\mathrm{EC}_{50}=8.25\right.$ and $8.69 \mu \mathrm{M}$, respectively). These activities were even greater when compared with those of benznidazole, the reference drug $\left(\mathrm{EC}_{50}=\right.$ $40.3 \mu \mathrm{M})[15]$. Triclosan and quinolone-hydrazone hybrids synthesized and evaluated in our group also exhibited good antitrypanosomal activity. Among these, hybrids $\mathbf{2 c}$ and $\mathbf{2 d}$ displayed the best results showing $\mathrm{EC}_{50}$ values of $1.10 \mu \mathrm{M}$ and $4.6 \mu \mathrm{M}$, respectively [16,17]. Quinoline-chalcone hybrid $2 \mathrm{e}$ also exhibited trypanocidal activity with a value of $31.73 \mu \mathrm{M}$ [18]. Furanchalcones-imidazole hybrids $2 \mathrm{f}$ $\left(\mathrm{EC}_{50}=0.66 \mu \mathrm{M}\right)$ and $\mathbf{2 g}\left(\mathrm{EC}_{50}=0.72 \mu \mathrm{M}\right)$, furanchalcone-chromone hybrid $\mathbf{2 h}\left(\mathrm{EC}_{50 \mathrm{i}}=13.78 \mu \mathrm{M}\right)$ and furanchalcone-quinoline hybrid $2 \mathbf{i}\left(\mathrm{EC}_{50^{-} \text {T.cruzi }}=7.09 \mu \mathrm{M}\right)$ also exhibited a good activity against $T$. cruzi [19]. Z. Qiao and coworkers have also investigated the biological action of hybrids such as chalconebenzoxaborole hybrid $\mathbf{2} \mathbf{j}$ which showed an $\mathrm{IC}_{50}$ of $0.01 \mu \mathrm{g} / \mathrm{mL}$ against bloodstream form of $T$. brucei and elimination of parasitemia in a murine model of infection [20]. On the other hand, M. A. Ismail and coworkers evaluated the in vitro biological action of biphenyl-benzimidazole-diamidines against Trypanosoma brucei rhodesiense, which showed $\mathrm{IC}_{50}$ values ranging from 3 to $37 \mathrm{nM}$, being $2 \mathbf{k}$ the most active compounds with an $\mathrm{IC}_{50}$ value of $3.0 \mathrm{nM}$ [21] (fig. 2). 
<smiles>O=C(/C=C/c1ccc(O)c(O)c1)OCCCOc1cc(Cl)ccc1Cc1ccc(Cl)cc1Cl</smiles><smiles>O=C(COc1cc(Cl)ccc1Cc1ccc(Cl)cc1Cl)N/N=C/c1ccccc1O</smiles><smiles>O=C(N/N=C/c1ccc(O)cc1O)c1ccc2ccccc2n1</smiles>

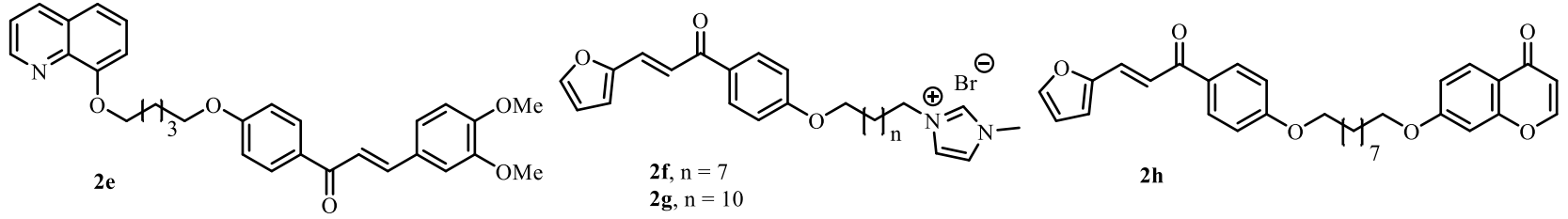<smiles>[Y1]CCOc1ccc(C(=O)/C=C/c2ccco2)cc1</smiles><smiles>COc1cc(C(=O)/C=C/c2ccc3c(c2)B(O)OC3)ccc1N</smiles><smiles>N=C(N)c1ccc2nc(-c3ccc(-c4ccc(C(=N)N)nc4)cc3)[nH]c2c1</smiles>

Fig. 2. Hybrids molecules derived from chalcone and biphenyl with antitrypanosomal activity

In the search for new therapeutic alternatives to treat Chagas disease, a series of furanchalconebiphenyl hybrids were designed, synthesized and evaluated in vitro as regards their cytotoxicity and anti-trypanosomal activity (fig. 3).
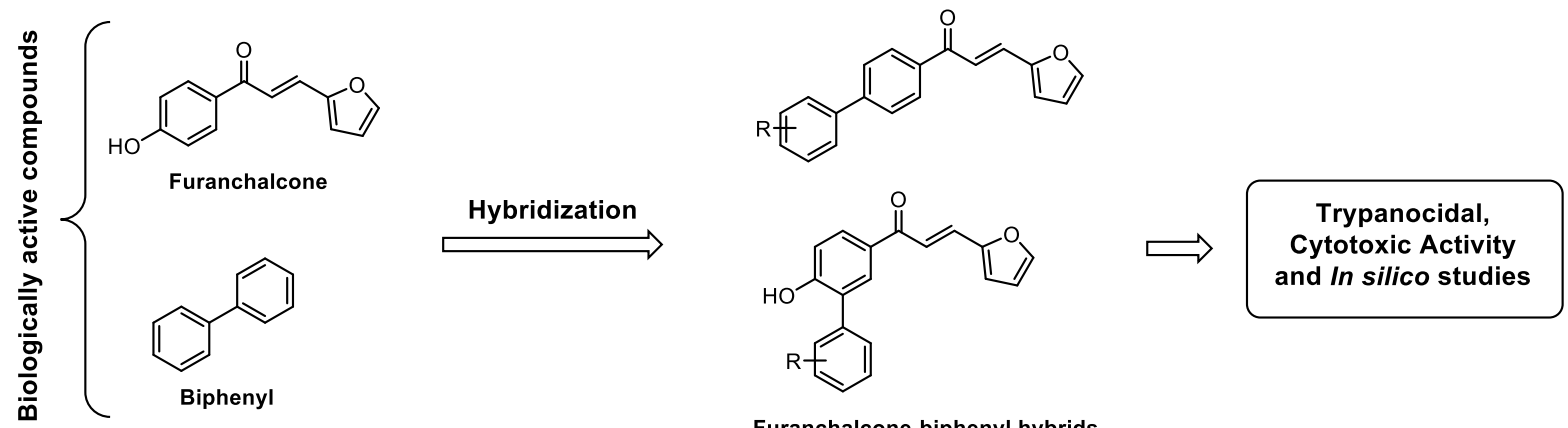

Furanchalcone-biphenyl hybrids

Fig. 3 Design of furanchalcone-biphenyl hybrids as antitrypanosomal agents

\section{Results and discussion}

\subsection{Chemistry}

The synthetic strategy for the preparation of biphenyl-furanchalcones is shown in Scheme 1. Thus, Claisen-Schmidt aldol condensation reaction of acetophenones $\mathbf{1}$ and $\mathbf{2}$ with furfural $\mathbf{3}$, yielded 
chalcones 4 and 5 (51\% and $85 \%$ yield), respectively [20]. Microwave assisted Suzuki reaction of furanchalcones 4 and 5 with boronic acids 6 a-i upon [22] afforded arylfuranchalcones $7 \mathbf{a}-\mathbf{i}$ and 8 a $-\mathbf{i}$ in $17-85 \%$. Compound $\mathbf{8 d}$ could not be obtained under the same reaction conditions.

The structure of each compound have been elucidated by a combined study of IR, ESI-MS, ${ }^{1} \mathrm{H}$ NMR, ${ }^{13} \mathrm{C}$ NMR and bidimensional analysis. The IR spectrum exhibits characteristic absorption peaks corresponding to $\mathrm{C}=\mathrm{O}, \mathrm{C}=\mathrm{C}, \mathrm{C}=\mathrm{C}_{\mathrm{Ar}}, \mathrm{C}-\mathrm{O}-\mathrm{C}, \mathrm{y} \mathrm{C}-\mathrm{H}_{\mathrm{Ar}}$. ESI-MS spectra exhibit characteristic $[\mathrm{M}+\mathrm{H}]^{+}$ peaks corresponding to their molecular weights. The assignation of all the signals to individuals $\mathrm{H}$ or $\mathrm{C}$ - atoms have been performed on the basis of typical $\delta$-values and J-constant coupling. ${ }^{1} \mathrm{H}-\mathrm{RMN}$ spectrum showed signal around 3.80-3.40 and 7.5 ppm corresponding to $-\mathrm{OCH}_{3}$ aryl group, $-\mathrm{C}=\mathrm{C}-\mathrm{H}$ furan ring and $-\mathrm{CH}=\mathrm{CH}-\mathrm{C}=\mathrm{O}$, respectively. The ${ }^{13} \mathrm{C}-\mathrm{NMR}$ spectrum showed signals of $\mathrm{C}=\mathrm{O}(\sim 187 \mathrm{ppm}), \mathrm{Ar}-$ $\mathrm{O}-(\sim 150-153 \mathrm{ppm})$, furan ring $(\sim 144 \mathrm{ppm}),-\mathrm{CH}=\mathrm{C}-\mathrm{C}=\mathrm{O}(\sim 130 \mathrm{ppm})$ and $-\mathrm{OCH}_{3}(\sim 56 \mathrm{ppm})$.

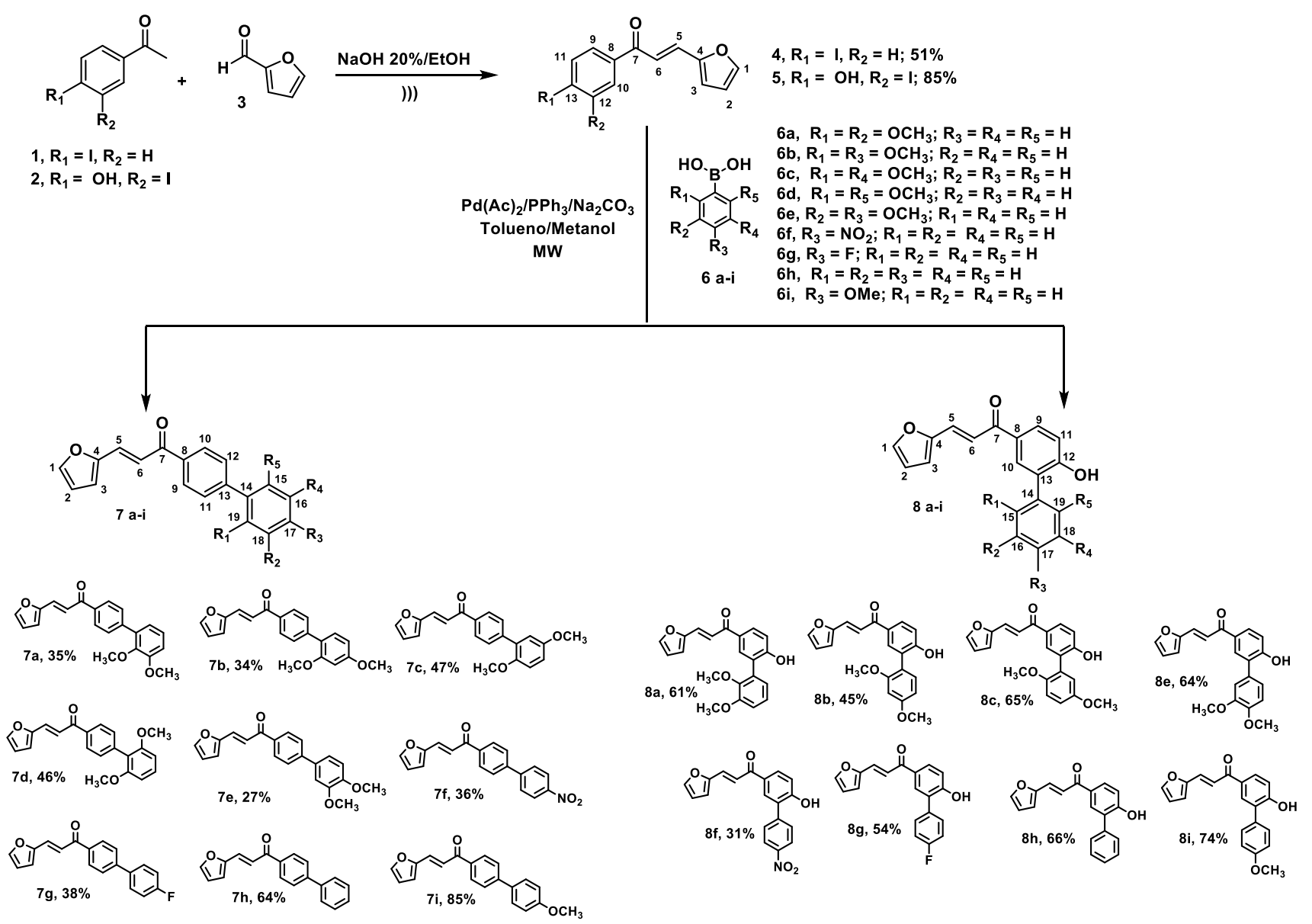

Scheme 1. Synthetic pathway to furanchalcone-biphenyl hybrids 


\subsection{Biological activities}

The effect of furanchalcone-biphenyl hybrids on cell growth and viability was assessed in human macrophages (U-937 cells) [23], which are the host cells for T. cruzi parasites. On the other hand, the antiparasite activity of these compounds was tested on intracellular amastigotes of $T$. cruzi [24,25], which are the parasite form causing the disease, assessing the ability of these compounds to reduce the amount of parasite living inside infected macrophages. Results are summarized in Table 1.

Table 1 In vitro cytotoxicity and antitrypanosomal activity of furanchalcone-biphenyl hybrids

\begin{tabular}{|c|c|c|c|}
\hline \multirow[t]{2}{*}{ Compound } & $\begin{array}{l}\text { Citotoxicity } \\
\text { (U-937 cells) }\end{array}$ & $\begin{array}{c}\text { Antitrypanosomal } \\
\text { activity }\end{array}$ & $\mathbf{S I}^{\mathrm{c}}$ \\
\hline & $\mathrm{LC}_{50}(\mathrm{Mean} \pm \mathrm{SEM})[\mu \mathrm{M}]^{\mathrm{a}}$ & $\mathrm{EC}_{50}(\mathrm{Mean} \pm \mathrm{SEM})[\mu \mathrm{M}]^{\mathrm{b}}$ & \\
\hline $7 \mathbf{a}$ & $16.34 \pm 0.12$ & $17.81 \pm 0.75$ & 0.92 \\
\hline $7 \mathrm{~b}$ & $15.82 \pm 0.15$ & $13.59 \pm 1.23$ & 1.16 \\
\hline $7 c$ & $15.81 \pm 0.39$ & $15.61 \pm 1.71$ & 1.01 \\
\hline 7d & $16.04+0.26$ & $15.71 \pm 1.86$ & 1.02 \\
\hline $7 e$ & $46.27 \pm 5.65$ & $83.01 \pm 14.23$ & 0.56 \\
\hline $7 f$ & $393.92 \pm 47.07$ & $86.94 \pm 6.22$ & 4.53 \\
\hline $7 \mathrm{~g}$ & $85.68 \pm 10.73$ & $99.71 \pm 5.76$ & 0.86 \\
\hline $7 \mathrm{~h}$ & $142.75 \pm 16.95$ & $259.66 \pm 48.0$ & 0.55 \\
\hline $7 \mathbf{i}$ & $283.36 \pm 102.64$ & $460.0 \pm 250.5$ & 0.61 \\
\hline $8 \mathbf{a}$ & $18.99 \pm 0.98$ & $18.15 \pm 0.54$ & 1.05 \\
\hline $8 \mathbf{b}$ & $19.44 \pm 1.40$ & $16.79 \pm 2.28$ & 1.16 \\
\hline $8 c$ & $15.65 \pm 0.17$ & $15.53 \pm 1.85$ & 1.01 \\
\hline $8 e$ & $15.61 \pm 0.32$ & $12.59 \pm 1.01$ & 1.24 \\
\hline
\end{tabular}




$\begin{array}{crrr}\mathbf{8 f} & 15.74 \pm 0.23 & \mathbf{1 0 . 5 2} \pm \mathbf{0 . 2 3} & 1.50 \\ \mathbf{8 g} & 17.77 \pm 0.46 & \mathbf{1 3 . 4 2} \pm \mathbf{0 . 3 9} & 1.32 \\ \mathbf{8 h} & 20.56 \pm 0.10 & 64.03 \pm 8.37 & 0.32 \\ \mathbf{8 i} & 17.54 \pm 0.22 & \mathbf{3 0 . 4 1} \pm \mathbf{5 . 4 6} & 0.58 \\ \mathbf{4} & 24.480 .98 & 15.460 .19 & 1.58 \\ \mathbf{5} & 17.740 .72 & 13.291 .04 & 1.34 \\ \text { Benznidazole } & 687.80 \pm 16.14 & 40.3 \pm 6.92 & 17.0\end{array}$

Data represent mean value $+/-$ standard deviation; ${ }^{a} \mathrm{LC}_{50}$ : Lethal Concentration 50 in $\mu \mathrm{M} ;{ }^{\mathrm{b}} \mathrm{EC}_{50}$ : Effective Concentration 50 in $\mu \mathrm{M} ;{ }^{\mathrm{c}} \mathrm{SI}$ : Selectivity Index $=\mathrm{LC}_{50} / \mathrm{EC}_{50}$.

Overall, all hybrids were highly cytotoxic to U-937 cells showing $\mathrm{LC}_{50}<200 \mu \mathrm{M}$ (Table 1). Compound 7f exhibited moderate cytotoxicity and benznidazole was not cytotoxic $\left(\mathrm{LC}_{50}>600 \mu \mathrm{M}\right)$. The antitrypanosomal activity was measured by determining the effective concentration $50\left(\mathrm{EC}_{50}\right)$ that corresponds to the concentration of drug that gives the half-maximal reduction of the amount of intracellular parasites (Table 1). Dose-response relationship showed that hybrids 7a-7d, 8a-8g, furanchalcone 4 and 5 were active against intracellular amastigotes of $T$. cruzi with $\mathrm{EC}_{50}$ of $<40 \mu \mathrm{M}$. The most active compounds were $\mathbf{7 b}$ and $\mathbf{8 e - 8 g}$ with an $\mathrm{EC}_{50}$ values of $13.59 \mu \mathrm{M}, 12.59 \mu \mathrm{M}, 10.52 \mu \mathrm{M}$ and $13.42 \mu \mathrm{M}$, respectively. In this case, benznidazole showed activity with an $\mathrm{EC}_{50}$ values of 40.3 $\mu \mathrm{M}$. The hybrids $\mathbf{7 b - 7 d}$ and $\mathbf{8 a - 8 g}$ showed better activity than reference drugs. Both the starting chalcones and the hybrids showed similar activities. This unexpected result could be due to the dominance of the mode of action of the chalcones, that could be explained by the presence of the Michael acceptor system that is reactive towards nucleophilic amino residues present in target enzymes of Leishmania [26,27]. Hybrids 7f, 8e, 8f, and $\mathbf{8 g}$ showed higher activity than cytotoxicity. Thus, the SI (Selectivity Index) values calculated for these compounds were $>1$ (Table 1). These results suggest that biological activity of these hybrids is selective, being more active against $T$. cruzi parasites than to U937 cells.

On a structure-activity relationship, it is interesting to note that electron withdrawing elements, such as the nitro group or fluorine atom, increase the activity $(7 \mathbf{g}, 7 \mathbf{f}$ vs $7 \mathbf{h}, 7 \mathbf{i}$ or $8 \mathrm{~g}, 8 \mathrm{f}$ vs $8 \mathbf{h}, \mathbf{8 i})$. In monosubstituted compounds series $(\mathbf{7 h}, 7 \mathbf{i}, 7 \mathbf{g}, 7 \mathbf{f}$ vs $8 \mathbf{h}, \mathbf{8 i}, \mathbf{8 g}, \mathbf{8 f})$ the presence of the hydroxyl group improves activity. This result is in agreement with the reports for several chalcones, coumarins, cinnamic ester and triclosan-caffeic acid hybrids [28-31]. The effect of the hydroxyl groups may be due 
to a better molecular recognition ability towards target bioreceptors upon hydrogen bond formation [32]. The degree of oxygenation is essential for activity, with dimethoxylated compounds (7b, 7e, 8b and 8e) exhibiting higher activity than monomethoxylated hybrids (7i and 8i). This result is in accordance with previous report [30]. Dimethoxylated compounds showed similar activity regardless the position of these oxygenated groups (7a-7e and 8a-8e). This result is inconclusive for these compounds. However, for the case of cinnamic esters we found that oxygenation in positions 3 and 4 of the phenyl group were fundamental for the activity [30], which could be associated with different mechanisms of action.

\subsection{Docking results}

Determining essential genes whose lost is not tolerable by the organism is a potential strategy to identify therapeutic targets in the development of anti-parasitic drugs. Cysteine proteases are essential for survival of both T. cruzi and T. brucei [33,34]. Among them, cruzipain have become a relevant protein target to design novel inhibitors for the treatment of Chagas disease [35]. This enzyme hydrolyzes chromogenic peptides at the carboxyl arginine or lysine terminus, and plays a key role in the development and differentiation of the parasite during various life cycle stages [36]. Chalcones are structurally related to classes of compounds that have been reported as novel potent cruzipain inhibitors, which can effectively cause the death of the parasite [37-39]. Therefore, it is of our interest to test in silico the affinity of the designed hybrids and to correlate the results with the experimental

findings. Thus, from docking experiments we examined the interactions between the hybrids and key residues of the protein (fig. 4). 
A.

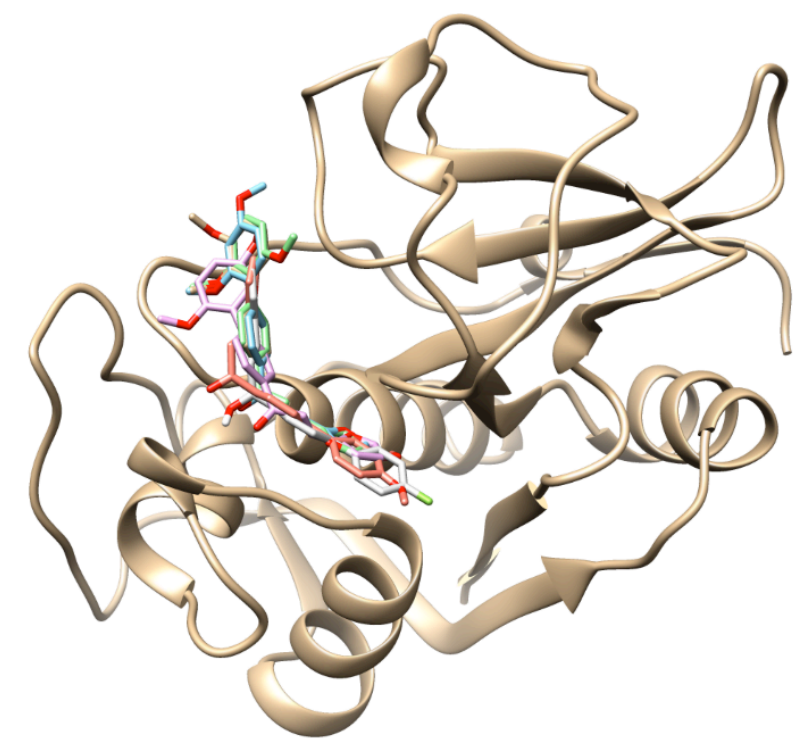

B.

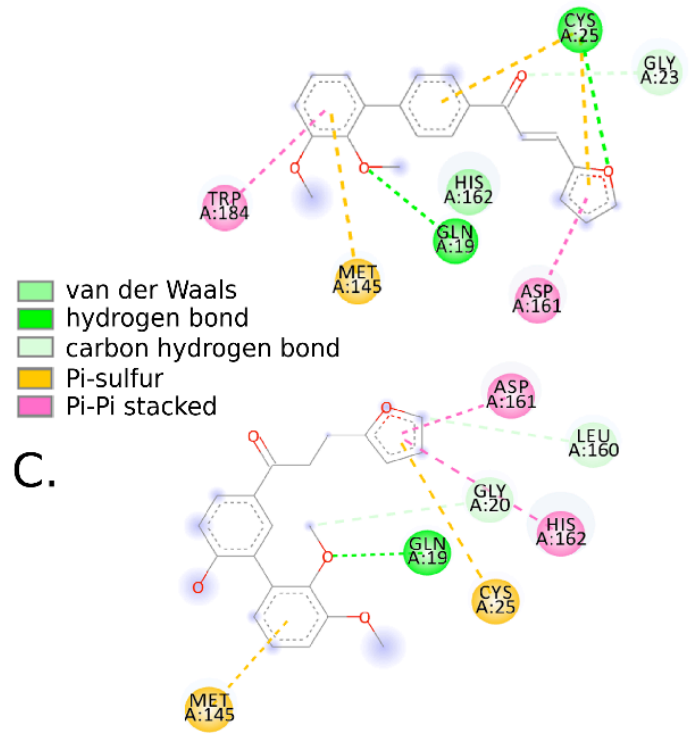

Fig 4. (A) Interaction of a set of hybrids (7 series) within the delimited active site of $T$. cruzi cruzipain structure (PDB:3I06). (B) 2D representation of interactions formed by compounds $7 \mathbf{a}$ and $7 \mathbf{g}$ with aminoacids of the enzyme.

In general, the compounds docked similarly across the delimited binding site, with a set of hydrophobic interactions that potentially confers stability during the binding event. The molecules also form diverse types of interactions, especially $\pi-\pi$ and $\pi$-sulfur between aromatic amino acids of the protein and ring motifs of the compounds. Table 2 summarizes the average scores per evaluated compound, and the corresponding spearman correlation factor.

Table 2 Average docking scores per compound evaluated and the spearman correlation against the average experimental binding data.

\begin{tabular}{cc}
\hline Compound & Average docking score (kcal/mol) \\
\hline $7 \mathrm{a}$ & -6.70 \\
$7 \mathrm{~b}$ & -6.94 \\
$7 \mathrm{c}$ & -6.72 \\
$7 \mathrm{~d}$ & -6.90 \\
$7 \mathrm{e}$ & -6.64 \\
$7 \mathrm{f}$ & -6.60 \\
$7 \mathrm{~g}$ & -6.82 \\
$7 \mathrm{~h}$ & -6.74 \\
$7 \mathrm{i}$ & -6.60 \\
$8 \mathrm{a}$ & -6.80 \\
$8 \mathrm{~b}$ & -6.70 \\
$8 \mathrm{c}$ & -6.82 \\
\hline
\end{tabular}




\begin{tabular}{cc}
\hline $8 \mathrm{e}$ & -6.96 \\
$8 \mathrm{f}$ & -7.10 \\
$8 \mathrm{~g}$ & -6.70 \\
$8 \mathrm{~h}$ & -6.70 \\
$8 \mathrm{i}$ & -6.80 \\
\hline Spearman correlation & $\mathbf{0 . 6 0 8}$ \\
\hline
\end{tabular}

According to the ranking correlation (which is higher than 0.5), cruzipain could be a potential candidate of the hybrids evaluated, despite the docking scores are close between each other in compound library with small structural differences. However, this initial hint can lead us to propose novel experimental evaluations against this molecular target, looking for optimizing the hits obtained in this project in a rational way. These findings are also supported by previous reports of active chalcones against this parasite protein target [40].

\subsection{Drug-Likeness Prediction Studies}

We calculated and analyzed various drug-likeness properties for the seventeen arylfuranchalcones derivatives and data are summarized in Table 3. All the compounds showed significant values for the parameters analyzed, exhibiting good drug like characteristics, besides, the values were found within the range of properties of $95 \%$ of currently known drugs. According to Lipinski's rule of five [41] (an orally active drug has no more than one violation of the criteria) the synthesized compounds $\mathbf{7 ( a - i )}$ and 8(a-i) could be orally active drugs in human. It was observed that all the title compounds exhibited good human intestinal absorption (\% HIA) and good percent of human oral absorption (\% HOA) ranging from 74.38 to $100 \%$. Greater HIA and HOA values denote that the synthetized compounds 7 (ai) and $\mathbf{8}(\mathbf{a}-\mathbf{i})$ could be better absorbed from the intestinal tract upon oral administration. These in silico ADMET predictions suggest that arylfuranchalcones derivatives $\mathbf{7 ( a - i )}$ and $\mathbf{8 ( a - i )}$ follow the criteria for orally active drugs and thus represent a pharmacologically active framework that should be considered on progressing further potential hits.

On the other hand, applicability of these physicochemical properties is modelling strategies for many processes, such as passive membrane permeation, where their molecular mechanism is hardly delineated and provides approaches such as structure-activity relationship (SAR). Furthermore, molecular polar surface area (PSA) is a descriptor that was shown to correlate well with passive molecular transport through membranes and allows prediction of drug-membrane interactions. A 
comparison of calculated PSA [42] values for 4-arylfuranchalcones $\mathbf{7}$ with the 3-arylfuranchalcones $\mathbf{8}$, displayed a significant deviation. Thus, when PSA was applied, a correlation between PSA values and $\mathrm{EC}_{50}$ numbers showed that a high PSA value (derivatives 8) favored their anti-trypanosomal activity, whereas low PSA value (compounds 7) contributed to a reduction of the antiprotozoal activity, suggesting that perhaps these polar compounds tend to have a greater affinity and good ability to penetrate through T. cruzi-infected cell.

On the other hand, lipophilicity is an important property of a drug molecule as it influences a number of physiological properties including transport through lipid bilayer. LogP gives a measure of the lipophilicity of a compound and it is a good indicator of permeability across the cell wall [43]. In this study, all tested compounds exhibited LogP values below 5, ranging from 2.887 to 4.773, suggesting good permeability and permeation across the cell membrane of infected cells. Additionally, in this work we calculated the number of rotatable bonds (Nrot), a topological parameter to measure flexibility, and we correlated this parameter with the anti-protozoal activity of the tested compounds 7(a-i) and 8(a-i). We found that the most active compounds exhibited an optimum antichagasic activity containing rotatable bonds in the range of 4 to 8 (see Table 3). This high conformational flexibility of the molecules suggests that all synthesized compounds display good absorption. Moreover, in silico artificial membrane permeation rate across Caco-2 cell monolayers or MDCK cell was calculated for all arylfuranchalcones derivatives. It was found that the passive transmembrane permeation of the novel compounds displayed high permeability values (from 720 to $5259 \mathrm{~nm} / \mathrm{sec}$ ), except for nitrosubstituted arylfuranchalcones $\mathbf{7 f}$ and $\mathbf{8 f}$ which displayed poor cell permeability values $(<400 \mathrm{~nm} / \mathrm{sec})$. In addition, early prediction of plasma protein binding ( $\left.\log \mathrm{K}_{\mathrm{HSA}}\right)$ has vital importance in the characterization of drug distribution in the systemic circulation. Unfavorable Log $\mathrm{K}_{\mathrm{HSA}}$ values can represent a negative effect on clinical development of promising drug candidates for human Chagas disease chemotherapy. Plasma protein binding affinity ( $\log \mathrm{K}_{\mathrm{HSA}}$ ) for the arylfuranchalcones derivatives displayed high binding affinity values (from -0.038 to 0.554 ). These values were very similar, up to $95 \%$, to known drugs (the recommended range for $95 \%$ of known drugs is -1.5 to 1.5 ), demonstrating the potential of these compounds as therapeutic candidates for the treatment of $T$. cruzi infection. 


\begin{tabular}{|c|c|c|c|c|c|c|c|c|c|c|c|c|c|c|}
\hline Entry & M.W = & D & $\underset{\left(7-200 A^{2}\right)}{\mathrm{PSA}^{\mathrm{b}}}$ & $\begin{array}{l}\text { n-Rot } \\
\text { Bond } \\
(<15)\end{array}$ & $\begin{array}{l}\mathrm{n}-\mathrm{QN}^{\mathrm{E}} \\
(=10)\end{array}$ & $\underset{(\infty)}{\mathrm{n}-\mathrm{OHNH}}$ & $\frac{\log P 0 / \underline{W^{5}}}{(-2.0-6.5)}$ & $\begin{array}{c}\log S^{f} \\
(-6-0.5)\end{array}$ & $\begin{array}{l}\log \mathrm{K}_{\mathrm{HSA}}{ }^{2} \\
(-1.5-1.2)\end{array}$ & $\begin{array}{c}\text { App. } \\
\text { Caco-2 } \\
(\mathrm{nm} / \mathrm{s}) \\
(<25 \text { poor }>500)\end{array}$ & $\begin{array}{c}\text { App. } \\
\text { MDCK } \\
(\mathrm{nm} / \mathrm{s})^{\mathrm{i}} \\
(<25 \text { poor })\end{array}$ & $\begin{array}{c}\% \\
\text { HIA }^{j}\end{array}$ & $\begin{array}{c}\text { Lipinski } \\
\text { Rule of } \\
\text { fuxe }(\measuredangle 1)\end{array}$ & $\begin{array}{c}\% \mathrm{HOA}^{\mathrm{k}} \\
(<\mathbf{2 5} \% \text { is } \\
\text { poor })\end{array}$ \\
\hline 4 & 324.117 & 4.563 & 35.460 & 4 & 2.25 & 0 & 3.509 & -3.690 & 0.044 & 3368 & 5259 & 96.77 & 0 & 100 \\
\hline 5 & 340.117 & 7.031 & 57.188 & 5 & 3.25 & 1 & 2.887 & -3.557 & -0.038 & 1201 & 1643 & 89.27 & 0 & 100 \\
\hline $7 a$ & 334.371 & 5.927 & 49.623 & 7 & 4.0 & 0 & 4.580 & -4.648 & 0.405 & 3419 & 1868 & 91.88 & 0 & 100 \\
\hline $7 b$ & 334.371 & 4.849 & 50.092 & 7 & 4.0 & 0 & 4.708 & -5.223 & 0.479 & 3365 & 1836 & 91.72 & 0 & 100 \\
\hline $7 c$ & 334.371 & 4.410 & 51.071 & 7 & 4.0 & 0 & 4.624 & -4.780 & 0.442 & 3380 & 1845 & 91.38 & 0 & 100 \\
\hline $7 d$ & 334.371 & 5.763 & 50.849 & 7 & 4.0 & 0 & 4.588 & -4.602 & 0.426 & 3369 & 1839 & 91.46 & 0 & 100 \\
\hline $7 \mathrm{e}$ & 334.371 & 4.068 & 51.069 & 7 & 4.0 & 0 & 4.639 & -5.135 & 0.452 & 3368 & 1838 & 91.38 & 0 & 100 \\
\hline $7 f$ & 319.316 & 6.912 & 80.314 & 6 & 3.5 & 0 & 3.783 & -4.893 & 0.411 & 403 & 185 & 81.29 & 0 & 96 \\
\hline $7 \mathrm{~g}$ & 292.309 & 4.436 & 35.473 & 5 & 2.5 & 0 & 4.767 & -5.210 & 0.554 & 3366 & 3297 & 96.76 & 0 & 100 \\
\hline $7 \mathrm{~h}$ & 274.318 & 4.379 & 36.292 & 5 & 2.5 & 0 & 4.474 & -4.647 & 0.472 & 3581 & 1964 & 96.48 & 0 & 100 \\
\hline $7 i$ & 304.345 & 5.311 & 44.774 & 6 & 3.25 & 0 & 4.483 & -4.695 & 0.426 & 3581 & 1964 & 93.55 & 0 & 100 \\
\hline $8 a$ & 350.370 & 8.252 & 69.510 & 8 & 4.75 & 1 & 4.042 & -4.465 & 0.338 & 1702 & 879 & 85.02 & 0 & 100 \\
\hline $8 \mathrm{~b}$ & 350.370 & 4.786 & 72.339 & 8 & 4.75 & 1 & 4.059 & -4.744 & 0.375 & 1440 & 734 & 84.04 & 0 & 100 \\
\hline $8 \mathrm{c}$ & 350.370 & 5.709 & 70.814 & 8 & 4.75 & 1 & 4.176 & -5.090 & 0.419 & 1427 & 726 & 84.57 & 0 & 100 \\
\hline $8 \mathrm{e}$ & 350.370 & 5.800 & 71.098 & 8 & 4.75 & 1 & 4.108 & -5.000 & 0.387 & 1505 & 769 & 84.47 & 0 & 100 \\
\hline $8 f$ & 335.315 & 11.85 & 100.35 & 7 & 4.25 & 1 & 4.773 & -5.347 & 0.334 & 167 & 71 & 74.38 & 0 & 86 \\
\hline $8 \mathrm{~g}$ & 308.308 & 7.230 & 55.497 & 6 & 3.25 & 1 & 4.148 & -4.958 & 0.418 & 1399 & 1277 & 89.85 & 0 & 100 \\
\hline $8 \mathrm{~h}$ & 290.318 & 3.096 & 57.639 & 6 & 3.25 & 1 & 3.937 & -4.569 & 0.379 & 1419 & 722 & 89.11 & 0 & 100 \\
\hline $8 \mathrm{i}$ & 320.344 & 3.105 & 66.121 & 7 & 4.0 & 1 & 3.987 & -4.711 & 0.373 & 1419 & 722 & 86.19 & 0 & 100 \\
\hline
\end{tabular}

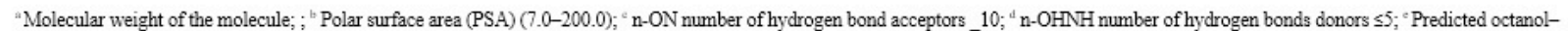
water partition coefficient $\left(\log\right.$ Po/w) $(-2.0$ to 6.5$)$; ${ }^{\prime}$ Logarithm of Aqueous solubility (Log S) $(-6$ to 0.5$) ;{ }^{8}$ Logarithm of predicted binding constant to human serum albumin (log $\left.\mathrm{K}_{\mathrm{HS}}\right)(-1.5$ to 1.2); "Predicted human intestinal permeability model (App. Caco-2); ; Apparent permeability across cellular membranes of Mladin-Darby canine kidney (MDCK) cells; ${ }^{-}$Human intestinal absorption $(\% \mathrm{HIA})\left(>80 \%\right.$ is high, $<25 \%$ is poor); ${ }^{k}$ percent of human oral absorption (HOA \%).

\section{Conclusions}

The synthesis, anti-trypanosomal and cytotoxic activities of seventeen furanchalcone derivatives are reported. This study showed that hybrids $\mathbf{7 a - 7 d}$ and $\mathbf{8 a - 8 g}$ were active against intracellular amastigotes of $T$. cruzi with $\mathrm{EC}_{50}$ of $<40 \mu \mathrm{M}$. The most active compounds were $\mathbf{7 b}$ and $\mathbf{8 e - 8 g}$ with an $\mathrm{EC}_{50}$ values of $13.59 \mu \mathrm{M}, 12.59 \mu \mathrm{M}, 10.52 \mu \mathrm{M}$ and $13.42 \mu \mathrm{M}$, respectively. The hybrids 7b-7d and 8a-8g exhibited better activity than reference drugs being compounds $\mathbf{7 f}, \mathbf{8 e}, \mathbf{8 f}$, and $\mathbf{8 g}$ the most selective ones. SAR analysis showed that electron withdrawing elements, such as the nitro group or fluorine atom, increase the activity. The degree of oxygenation is essential for activity being dimethoxylated compounds, regardless the position of these oxygenated groups, more active than monomethoxylated hybrids. In silico ADMET studies of arylfuranchalcones derivatives $\mathbf{7 ( a - i )}$ and $\mathbf{8}(\mathbf{a}-\mathbf{i})$, showed that these novel compounds have good drug like properties, making them potentially promising agents for antichagasic therapy. Physicochemical and ADMET profile of these molecules, such as polar surface area (PSA), LogP and the number of rotatable bonds (Nrot), Log P (se repite ????), membrane permeation rate and Plasma Protein Binding ( $\log \mathrm{K}_{\mathrm{HSA}}$ ) showed that these compounds have potential for an eventual development as oral agents and can be significant active drug candidates in search of better and safe anti-trypanosomal agents. The structural analysis allowed us to determine whether cruzipain could be a 
potential molecular target of the evaluated compounds. Among the cysteine proteases reported in $T$. cruzi, cruzipain is a key protein that has been studied for inhibition purposes with different molecules, including chalcones. In our case, we found a significant prediction correlation with this enzyme, providing us clues for further optimization steps of the synthesized hybrids. This study has showed that these furanchalcone-byphenyl hybrids have potential to be considered as candidates for antitrypanosomal drug development.

\section{Experimental section}

\subsection{Chemical synthesis}

\subsubsection{General remarks}

Microwave reactions were carried out in a CEM Discover microwave reactor in sealed vessels (monowave, maximum power $300 \mathrm{~W}$, temperature control by IR sensor, fixed temperature). ${ }^{1} \mathrm{H}$ and ${ }^{13} \mathrm{C}$ NMR spectra were recorded on a Varian instrument operating at 300 and $75 \mathrm{MHz}$, respectively. The signals of the deuterated solvent $\left(\mathrm{CDCl}_{3}\right.$ or $\left.\mathrm{CD}_{3} \mathrm{OD}\right)$ were used as reference $\left(\mathrm{CDCl}_{3}: \delta=7.27\right.$ ppm for ${ }^{1} \mathrm{H}$ NMR and $\delta=77.00$ ppm for ${ }^{13} \mathrm{C}$ NMR; $\mathrm{CD}_{3} \mathrm{OD}: \delta=3.31$ and $4.87 \mathrm{ppm}$ for ${ }^{1} \mathrm{H}$ NMR and $\delta=49.2$ ppm for $\left.{ }^{13} \mathrm{C} \mathrm{NMR}\right)$. Carbon atom types $\left(\mathrm{C}, \mathrm{CH}, \mathrm{CH}_{2}, \mathrm{CH}_{3}\right)$ were determined by using the DEPT or APT pulse sequence. Signal were assigned using two dimensional heteronuclear correlations (COSY, HSQC and HMBC). High resolution mass spectra were recorded using electrospray ionization mass spectrometry (ESI-MS). A QTOF Premier instrument with an orthogonal Z-spray-electrospray interface (Waters, Manchester, UK) was used operating in the W-mode. The drying and cone gas was nitrogen set to flow rates of 300 and $30 \mathrm{~L} / \mathrm{h}$, respectively. Methanol sample solutions (ca. 1 x $10^{-5} \mathrm{M}$ ) were directly introduced into the ESI spectrometer at a flow rate of $10 \mu \mathrm{L} / \mathrm{min}$. A capillary voltage of $3.5 \mathrm{kV}$ was used in the positive scan mode, and the cone voltage set to Uc $=10 \mathrm{~V}$. For accurate mass measurements, a $2 \mathrm{mg} / \mathrm{L}$ standard solution of leucine enkephalin was introduced via the lock spray needle at a cone voltage set to $85 \mathrm{~V}$ and a flow rate of $30 \mu \mathrm{L} / \mathrm{min}$. IR spectra were recorded on a Spectrum RX I FT-IR system (Perkin-Elmer, Waltham, MA, USA) in KBr disks. Silica gel 60 (0.0630.200 mesh, Merck, Whitehouse Station, NJ, USA) was used for column chromatography, and precoated silica gel plates (Merck 60 F254 $0.2 \mathrm{~mm}$ ) were used for thin layer chromatography (TLC). 
4.1.2. General procedure for the synthesis of furanchalcones $(\mathbf{4 , 5})$

Acetophenones 1 or $\mathbf{2}$ (1 eq) and furfural 3 (1.1 eq) were added to an ethanolic solution of $\mathrm{NaOH}$ $20 \%$. The mixture was sonicated for 45 minutes and neutralized with a solution $\mathrm{HCl} 10 \%$ in an ice bath. The yellow solid was filtered, washed with water and dried. The solid was recrystallized with methanol to get the furanochalcones 4 and $\mathbf{5}$ with yields of $51 \%$ an $85 \%$ respectively. Monitoring the reaction progress and product purification was carried by TLC.

4.1.2.1. (2E)-3-(furan-2-il)-1-(4-iodofenil)prop-2-en-1-ona (4): Yield 51\%; pale yellow solid; m.p. 61$64^{\circ} \mathrm{C}$; IR $\left(\mathrm{cm}^{-1}\right): v_{\max } 1658(\mathrm{C}=\mathrm{O}), 1583(\mathrm{C}=\mathrm{C}), 1543\left(\mathrm{C}=\mathrm{C}_{\mathrm{Ar}}\right), 995\left(-\mathrm{C}-\mathrm{O}-\mathrm{C}_{\text {Furane }}\right), 804\left(\mathrm{C}-\mathrm{H}_{\mathrm{Ar}}\right) .{ }^{1} \mathrm{H}-$ $\operatorname{NMR}\left(\mathrm{CDCl}_{3}, 300 \mathrm{MHz}\right): \delta 6.57\left(\mathrm{H}_{2}, \mathrm{dd}, J 1=1.70, J 2=3.23 \mathrm{~Hz}\right), 6.73\left(\mathrm{H}_{3}, \mathrm{~d}, J=3.23 \mathrm{~Hz}\right), 7.38\left(\mathrm{H}_{5}\right.$, d, $J=15.32 \mathrm{~Hz}), 7.53\left(\mathrm{H}_{1}, \mathrm{~s}_{\text {apparent }}\right), 7.59\left(\mathrm{H}_{6}, \mathrm{~d}, J=15.32 \mathrm{~Hz}\right), 7.73\left(\mathrm{H}_{9}, \mathrm{H}_{10}, \mathrm{~d}, J=8.45 \mathrm{~Hz}\right), 7.85$ $\left(\mathrm{H}_{11}, \mathrm{H}_{12}, \mathrm{~d}, J=8.45 \mathrm{~Hz}\right) ;{ }^{13} \mathrm{C}-\mathrm{NMR}\left(\mathrm{CDCl}_{3}, 75 \mathrm{MHz}\right): \delta 100.68\left(\mathrm{C}_{13}\right), 112.84\left(\mathrm{C}_{2}\right), 116.78\left(\mathrm{C}_{3}\right)$, $118.60\left(\mathrm{C}_{6}\right), 129.88\left(\mathrm{C}_{9}, \mathrm{C}_{10}\right), 131.15\left(\mathrm{C}_{5}\right), 137.44\left(\mathrm{C}_{8}\right), 137.94\left(\mathrm{C}_{11}, \mathrm{C}_{12}\right), 145.18\left(\mathrm{C}_{1}\right), 151.56\left(\mathrm{C}_{4}\right)$, $188.97\left(\mathrm{C}_{7}\right)$. ESI-MS: m/z $324.9725[\mathrm{M}+\mathrm{H}]^{+}$, Calc. for $\mathrm{C}_{13} \mathrm{H}_{9} \mathrm{IO}_{2}: 324.9726$

4.1.2.2. (2E)-3-(furan-2-il)-1-(4-hidroxi-3-iodofenil)prop-2-en-1-ona (5): Yield 85\%; yellow solid; m.p 169-170 ${ }^{\circ} \mathrm{C}$; IR $\left(\mathrm{cm}^{-1}\right)$ : $v_{\max } 3369(-\mathrm{OH}), 1629(\mathrm{C}=\mathrm{O}), 1579(\mathrm{C}=\mathrm{C}), 1519\left(\mathrm{C}=\mathrm{C}_{\mathrm{Ar}}\right), 1014(-\mathrm{C}-\mathrm{O}-\mathrm{C}-$

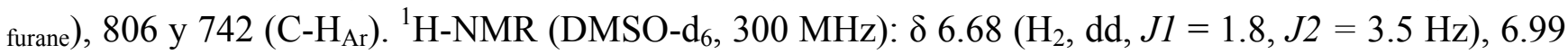
$\left(\mathrm{H}_{12}, \mathrm{~d}, J=8.50 \mathrm{~Hz}\right), 7.09\left(\mathrm{H}_{3}, \mathrm{~d}, J=3.5 \mathrm{~Hz}\right), 7.52\left(\mathrm{H}_{5}, \mathrm{H}_{1}, \mathrm{~s}_{\text {apparent }}\right), 7.90\left(\mathrm{H}_{6}, \mathrm{~s}\right), 7.99\left(\mathrm{H}_{9}, \mathrm{dd}, J 1=\right.$ $2.05, J 2=8.5 \mathrm{~Hz}), 8.38\left(\mathrm{H}_{10}, \mathrm{~d}, J=2.05 \mathrm{~Hz}\right) ;{ }^{13} \mathrm{C}-\mathrm{NMR}\left(\mathrm{DMSO}_{6}, 75 \mathrm{MHz}\right): \delta 85.63\left(\mathrm{C}_{11}\right), 113.55$ $\left(\mathrm{C}_{2}\right), 115.10\left(\mathrm{C}_{12}\right), 117.14\left(\mathrm{C}_{3}\right), 118.89\left(\mathrm{C}_{6}\right), 130.40\left(\mathrm{C}_{9}\right), 131.14\left(\mathrm{C}_{5}\right), 131.19\left(\mathrm{C}_{8}\right), 140.10\left(\mathrm{C}_{10}\right)$, $146.52\left(\mathrm{C}_{1}\right), 151.69\left(\mathrm{C}_{4}\right), 161.62\left(\mathrm{C}_{13}\right), 186.01\left(\mathrm{C}_{7}\right)$. ESI-MS: $m / z \quad 294.9884[\mathrm{M}+\mathrm{H}]^{+}$, Calc. for $\mathrm{C}_{13} \mathrm{H}_{9} \mathrm{IO}_{3}: 294.9888$

\subsubsection{General procedure for the synthesis of aryl-furanchalcones (7a-7i and $\mathbf{8 a - 8 i})$}

A mixture of 4-iodofuranochalcona 4 or 4-hydroxy-3-iodofuranochalcona 5 (1 eq), boronic acid 6 a - i (2 eq), palladium acetate (5\%), triphenylphosphine $(10 \%)$, sodium carbonate (7eq) and $4 \mathrm{~mL}$ of toluene:methanol (3:1), were heated under microwave radiation for 40 minutes $\left(100^{\circ} \mathrm{C}, 200 \mathrm{~W}\right)$. then the mixture were diluted with dichloromethane and filtered. The crude reaction mixture, was evaporated under reduced pressure and the residue was purified by preparative TLC eluting with a mixture of dichlorometahane:hexanes (9:1). The final product was recrystallized to obtain the arylfuranochalcones $7 \mathbf{a}-\mathbf{i}$ and $\mathbf{8} \mathbf{a}-\mathbf{i}$ with yield between $27-85 \%$ y $61-65 \%$ respectively. 
4.1.3.1. (2E)-1-\{2',3'-dimethoxy-[1,1'-biphenyl]-4-yl\}-3-(furan-2-iy)prop-2-en-1-one (7a): Yield 35\%; beige solid; m.p. $111-114{ }^{\circ} \mathrm{C}$; IR $\left(\mathrm{cm}^{-1}\right)$ : $v_{\max } 2933\left(-\mathrm{CH}_{3}\right), 1666(\mathrm{C}=\mathrm{O}), 1597(\mathrm{C}=\mathrm{C}), 1548\left(\mathrm{C}=\mathrm{C}_{\mathrm{Ar}}\right)$, 1263 y $1035\left(-\mathrm{OCH}_{3} \mathrm{Ar}\right), 1006$ (-C-O-C- furane), 796, 736 y $700\left(\mathrm{C}-\mathrm{H}_{\mathrm{Ar}}\right) .{ }^{1} \mathrm{H}-\mathrm{NMR}\left(\mathrm{CDCl}_{3}, 300 \mathrm{MHz}\right): \delta$ $3.60\left(-\mathrm{O}-\mathrm{CH}_{3}, \mathrm{~s}\right), 3.93\left(-\mathrm{O}-\mathrm{CH}_{3}, \mathrm{~s}\right), 6.53\left(\mathrm{H}_{2}, \mathrm{dd}, J 1=3.4\right.$ y $\left.J 2=1.8\right), 6.74\left(\mathrm{H}_{3}, \mathrm{~d}, J=3.02 \mathrm{~Hz}\right), 6.93-$ $7.02\left(\mathrm{H}_{15}, \mathrm{H}_{17}, \mathrm{~m}\right), 7.15\left(\mathrm{H}_{16}, \mathrm{t}, J=7.88 \mathrm{~Hz}\right), 7.52\left(\mathrm{H}_{1}, \mathrm{H}_{6}, \mathrm{~d}, J=15.50 \mathrm{~Hz}\right), 7.63\left(\mathrm{H}_{5}, \mathrm{~d}, J=15.50 \mathrm{~Hz}\right)$, $7.70\left(\mathrm{H}_{11}, \mathrm{H}_{12}, \mathrm{~d}, J=8.20 \mathrm{~Hz}\right), 8.09\left(\mathrm{H}_{9}, \mathrm{H}_{10}, \mathrm{~d}, J=8.20 \mathrm{~Hz}\right) .{ }^{13} \mathrm{C}-\mathrm{NMR}\left(\mathrm{CDCl}_{3}, 300 \mathrm{MHz}\right): \delta 56.00(-$ $\left.\mathrm{O}-\underline{\mathrm{CH}}_{3}\right), 60.80\left(-\mathrm{O}-\underline{\mathrm{CH}_{3}}\right), 112.24\left(\mathrm{C}_{17}\right), 112.71\left(\mathrm{C}_{2}\right), 116.25\left(\mathrm{C}_{3}\right), 119.38\left(\mathrm{C}_{6}\right), 122.40\left(\mathrm{C}_{15}\right), 124.31$ $\left(\mathrm{C}_{16}\right), 128.36\left(\mathrm{C}_{11}, \mathrm{C}_{12}\right), 129.59\left(\mathrm{C}_{9}, \mathrm{C}_{10}\right), 130.59\left(\mathrm{C}_{5}\right), 134.88\left(\mathrm{C}_{14}\right), 136.77\left(\mathrm{C}_{14}\right), 142.94\left(\mathrm{C}_{8}\right), 144.94$ $\left(\mathrm{C}_{1}\right), 146.65\left(\mathrm{C}_{19}\right), 151.78\left(\mathrm{C}_{4}\right), 153.23\left(\mathrm{C}_{18}\right), 189.54\left(\mathrm{C}_{7}\right)$. ESI-MS: $m / z$ 335.1281 [M + H] $]^{+}$, Calc. for $\mathrm{C}_{21} \mathrm{H}_{18} \mathrm{O}_{4}: 335.1283$

4.1.3.2. (2E)-1-\{2',4'-dimethoxy-[1,1'-biphenyl]-4-yl\}-3-(furan-2-iy)prop-2-en-1-one (7b): Yield 34\%; yellow pale solid; m.p. 114-117 ${ }^{\circ} \mathrm{C}$; IR $\left(\mathrm{cm}^{-1}\right): v_{\max } 2927\left(-\mathrm{CH}_{3}\right), 1651(\mathrm{C}=\mathrm{O}), 1583(\mathrm{C}=\mathrm{C}), 1548$

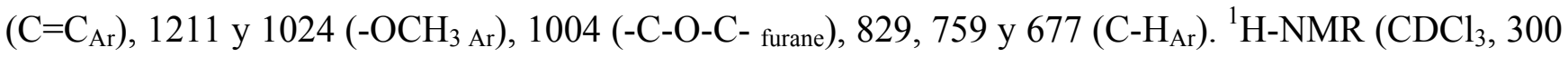
$\mathrm{MHz}): \delta 3.82\left(-\mathrm{O}-\underline{\mathrm{C}}_{3}, \mathrm{~s}\right), 3.86\left(-\mathrm{O}-\underline{\mathrm{C}}_{3}, \mathrm{~s}\right), 6.52\left(\mathrm{H}_{2}, \mathrm{dd}, J 1=1.8\right.$ y $\left.J 2=3.3 \mathrm{~Hz}\right), 6.55-6.63\left(\mathrm{H}_{16}, \mathrm{H}_{18}\right.$, m), $6.72\left(\mathrm{H}_{3}, \mathrm{~d}, J=3.30 \mathrm{~Hz}\right), 7.29\left(\mathrm{H}_{19}, \mathrm{~d}, J=8.11 \mathrm{~Hz}\right), 7.51\left(\mathrm{H}_{6}, \mathrm{H}_{5}, \mathrm{~d}, J=14.99 \mathrm{~Hz}\right), 7.60\left(\mathrm{H}_{1}\right.$, Sapparent), $7.64\left(\mathrm{H}_{11}, \mathrm{H}_{12}, \mathrm{~d}, J=8.23 \mathrm{~Hz}\right), 8.07\left(\mathrm{H}_{9}, \mathrm{H}_{10}, \mathrm{~d}, J=8.23 \mathrm{~Hz}\right) ;{ }^{13} \mathrm{C}-\mathrm{NMR}\left(\mathrm{CDCl}_{3}, 75 \mathrm{MHz}\right): \delta$ $55.50\left(-\mathrm{O}-\underline{\mathrm{CH}_{3}}\right), 55.60\left(-\mathrm{O}-\underline{\mathrm{C}} \mathrm{H}_{3}\right), 99.07\left(\mathrm{C}_{16}\right), 104.90\left(\mathrm{C}_{18}\right), 112.69\left(\mathrm{C}_{2}\right), 116.09\left(\mathrm{C}_{3}\right), 119.46\left(\mathrm{C}_{6}\right)$, $122.38\left(\mathrm{C}_{19}\right), 128.30\left(\mathrm{C}_{11}, \mathrm{C}_{12}\right), 129.61\left(\mathrm{C}_{9}, \mathrm{C}_{10}\right), 130.40\left(\mathrm{C}_{14}\right), 131.35\left(\mathrm{C}_{5}\right), 136.06\left(\mathrm{C}_{13}\right), 143.24\left(\mathrm{C}_{8}\right)$, $144.86\left(\mathrm{C}_{1}\right), 151.83\left(\mathrm{C}_{4}\right), 157.64\left(\mathrm{C}_{15}\right), 161.00\left(\mathrm{C}_{17}\right), 189.39\left(\mathrm{C}_{7}\right)$. ESI-MS: m/z $335.1278[\mathrm{M}+\mathrm{H}]^{+}$, Calc. for $\mathrm{C}_{21} \mathrm{H}_{18} \mathrm{O}_{4}: 335.1283$.

4.1.3.3. (2E)-1-\{2',5'-dimethoxy-[1,1'-biphenyl]-4-yl\}-3-(furan-2-yl)prop-2-en-1-one (7c): Yield 47\%; yellow pale solid; m.p. 87-90 ${ }^{\circ} \mathrm{C}$; IR $\left(\mathrm{cm}^{-1}\right): v_{\max } 2935\left(-\mathrm{CH}_{3}\right), 1662(\mathrm{C}=\mathrm{O}), 1604(\mathrm{C}=\mathrm{C}), 1552$ $\left(\mathrm{C}=\mathrm{C}_{\mathrm{Ar}}\right), 1220$ y $1047\left(-\mathrm{OCH}_{3} \mathrm{Ar}\right), 1016(-\mathrm{C}-\mathrm{O}-\mathrm{C}-$ furane$), 723\left(\mathrm{C}-\mathrm{H}_{\mathrm{Ar}}\right) .{ }^{1} \mathrm{H}-\mathrm{NMR}\left(\mathrm{CDCl}_{3}, 300 \mathrm{MHz}\right): \delta$ $3.77\left(-\mathrm{O}-\mathrm{C}_{3}, \mathrm{~s}\right), 3.82\left(-\mathrm{O}-\underline{\mathrm{CH}}_{3}, \mathrm{~s}\right), 6.52\left(\mathrm{H}_{2}, \mathrm{dd}, J 1=1.8\right.$ y $\left.J 2=3.4 \mathrm{~Hz}\right), 6.73\left(\mathrm{H}_{3}, \mathrm{~d}, J=3.40 \mathrm{~Hz}\right)$, 6.83-6.98 $\left(\mathrm{H}_{6}, \mathrm{H}_{17}, \mathrm{H}_{18}, \mathrm{~m}\right), 7.51\left(\mathrm{H}_{5}, \mathrm{H}_{15}, \mathrm{~d}, J=15.66 \mathrm{~Hz}\right), 7.60\left(\mathrm{H}_{1}, \mathrm{~s}\right), 7.67\left(\mathrm{H}_{11}, \mathrm{H}_{12}, \mathrm{~d}, J=8.4 \mathrm{~Hz}\right)$, $8.08\left(\mathrm{H}_{9}, \mathrm{H}_{10}, \mathrm{~d}, J=8.4 \mathrm{~Hz}\right) ;{ }^{13} \mathrm{C}-\mathrm{NMR}\left(\mathrm{CDCl}_{3}, 75 \mathrm{MHz}\right): \delta 55.87\left(-\mathrm{O}-\underline{\mathrm{CH}}_{3}\right), 56.34\left(-\mathrm{O}-\underline{\mathrm{CH}}_{3}\right), 112.71$ $\left(\mathrm{C}_{18}\right), 112.79\left(\mathrm{C}_{2}\right), 113.94\left(\mathrm{C}_{17}\right), 116.21\left(\mathrm{C}_{15}\right), 116.62\left(\mathrm{C}_{3}\right), 119.40\left(\mathrm{C}_{6}\right), 128.29\left(\mathrm{C}_{9}, \mathrm{C}_{10}\right), 129.76\left(\mathrm{C}_{11}\right.$, $\left.\mathrm{C}_{12}\right), 130.49\left(\mathrm{C}_{5}\right), 130.57\left(\mathrm{C}_{14}\right), 136.66\left(\mathrm{C}_{13}\right), 143.13\left(\mathrm{C}_{8}\right), 144.92\left(\mathrm{C}_{1}\right), 150.82\left(\mathrm{C}_{4}\right), 151.79\left(\mathrm{C}_{19}\right)$, $153.84\left(\mathrm{C}_{16}\right), 189.46\left(\mathrm{C}_{7}\right)$. ESI-MS: $m / z$ 335.1277 [M+ H] $]^{+}$, Calc. for $\mathrm{C}_{21} \mathrm{H}_{18} \mathrm{O}_{4}: 335.1283$. 
4.1.3.4. (2E)-1-\{2',6'-dimethoxy-[1,1'-biphenyl]-4-yl\}-3-(furan-2-yl)prop-2-en-1-one (7d): Yield 46\%; beige solid; m.p. $140-143{ }^{\circ} \mathrm{C}$; IR $\left(\mathrm{cm}^{-1}\right)$ : $v_{\max } 2927\left(-\mathrm{CH}_{3}\right), 1652(\mathrm{C}=\mathrm{O}), 1587(\mathrm{C}=\mathrm{C}), 1550\left(\mathrm{C}=\mathrm{C}_{\mathrm{Ar}}\right)$, 1246 y $1103\left(-\mathrm{OCH}_{3 \mathrm{Ar}}\right), 1012$ (-C-O-C- furane), $732\left(\mathrm{C}-\mathrm{H}_{\mathrm{Ar}}\right) .{ }^{1} \mathrm{H}-\mathrm{NMR}\left(\mathrm{CDCl}_{3}, 300 \mathrm{MHz}\right): \delta 3.75$ (-O$\left.\mathrm{CH}_{3}, \mathrm{~s}\right), 6.52\left(\mathrm{H}_{2}, \mathrm{dd}, J 1=1.8\right.$ y $\left.J 2=3.40 \mathrm{~Hz}\right), 6.67\left(\mathrm{H}_{16}, \mathrm{H}_{18}, \mathrm{~d}, J=8.40 \mathrm{~Hz}\right), 6.72\left(\mathrm{H}_{3}, \mathrm{~d}, J=3.40\right.$ $\mathrm{Hz}), 7.32\left(\mathrm{H}_{17}, \mathrm{t}, J=8.40 \mathrm{~Hz}\right), 7.46-7.57\left(\mathrm{H}_{1}, \mathrm{H}_{6}, \mathrm{H}_{11}, \mathrm{H}_{12}, \mathrm{~m}\right), 7.62\left(\mathrm{H}_{5}, \mathrm{~d}, J=15.30 \mathrm{~Hz}\right), 8.08\left(\mathrm{H}_{9}\right.$, $\left.\mathrm{H}_{10}, \mathrm{~d}, J=8.40 \mathrm{~Hz}\right) ;{ }^{13} \mathrm{C}-\mathrm{NMR}\left(\mathrm{CDCl}_{3}, 75 \mathrm{MHz}\right): \delta 55.94\left(-\mathrm{O}-\underline{\mathrm{C}}_{3}\right), 104.22\left(\mathrm{C}_{16}, \mathrm{C}_{18}\right), 112.65\left(\mathrm{C}_{2}\right)$, $116.00\left(\mathrm{C}_{3}\right), 118.44\left(\mathrm{C}_{6}\right), 119.55\left(\mathrm{C}_{13}\right), 127.90\left(\mathrm{C}_{11}, \mathrm{C}_{12}\right), 129.38\left(\mathrm{C}_{5}\right), 130.35\left(\mathrm{C}_{17}\right), 131.39\left(\mathrm{C}_{9}, \mathrm{C}_{10}\right)$, $136.41\left(\mathrm{C}_{14}\right), 139.45\left(\mathrm{C}_{8}\right), 144.81\left(\mathrm{C}_{1}\right), 151.86\left(\mathrm{C}_{4}\right), 157.55\left(\mathrm{C}_{15}, \mathrm{C}_{19}\right), 189.58\left(\mathrm{C}_{7}\right)$. ESI-MS: $\mathrm{m} / \mathrm{z}$ $335.1285[\mathrm{M}+\mathrm{H}]^{+}$, Calc. for $\mathrm{C}_{21} \mathrm{H}_{18} \mathrm{O}_{4}: 335.1283$.

4.1.3.5. (2E)-1-\{3',4'-dimethoxy-[1,1'-biphenyl]-4-yl\}-3-(furan-2-yl)prop-2-en-1-one (7e): Yield 27\%; yellow solid; m.p. $131-134{ }^{\circ} \mathrm{C}$; IR $\left(\mathrm{cm}^{-1}\right)$ : v $\max 2951\left(-\mathrm{CH}_{3}\right), 1654(\mathrm{C}=\mathrm{O}), 1595(\mathrm{C}=\mathrm{C}), 1548\left(\mathrm{C}=\mathrm{C}_{\mathrm{Ar}}\right)$, 1222 y $1147\left(-\mathrm{OCH}_{3} \mathrm{Ar}\right), 1016$ (-C-O-C- furane), 806 y $754\left(\mathrm{C}-\mathrm{H}_{\mathrm{Ar}}\right) .{ }^{1} \mathrm{H}-\mathrm{NMR}\left(\mathrm{CDCl}_{3}, 300 \mathrm{MHz}\right): \delta 3.94$ (-O-C $\left.\underline{\mathrm{H}}_{3}, \mathrm{~s}\right), 3.98\left(-\mathrm{O}-\mathrm{C}_{3}, \mathrm{~s}\right), 6.53\left(\mathrm{H}_{2}, \mathrm{~s}_{\text {apparent }}\right), 6.74\left(\mathrm{H}_{3}, \mathrm{~d}, J=3.30 \mathrm{~Hz}\right), 6.97\left(\mathrm{H}_{16}, \mathrm{~d}, J=8.40 \mathrm{~Hz}\right)$, $7.18\left(\mathrm{H}_{5}, \mathrm{H}_{15}, \mathrm{~d}, J=15.54 \mathrm{~Hz}\right), 7.50\left(\mathrm{H}_{6}, \mathrm{~d}, J=15.54 \mathrm{~Hz}\right), 7.55\left(\mathrm{H}_{19}, \mathrm{~s}\right), 7.61\left(\mathrm{H}_{1}, \mathrm{~s}\right), 7.69\left(\mathrm{H}_{11}, \mathrm{H}_{12}, \mathrm{~d}, J\right.$ $=8.20 \mathrm{~Hz}), 8.10\left(\mathrm{H}_{9}, \mathrm{H}_{10}, \mathrm{~d}, J=8.20 \mathrm{~Hz}\right) ;{ }^{13} \mathrm{C}-\mathrm{NMR}\left(\mathrm{CDCl}_{3}, 75 \mathrm{MHz}\right): \delta 56.05\left(-\mathrm{O}-\underline{\mathrm{C}}_{3}\right), 110.38$ $\left(\mathrm{C}_{19}\right), 111.54\left(\mathrm{C}_{16}\right), 112.74\left(\mathrm{C}_{2}\right), 116.30\left(\mathrm{C}_{3}\right), 119.27\left(\mathrm{C}_{6}\right), 119.86\left(\mathrm{C}_{15}\right), 126.89\left(\mathrm{C}_{11}, \mathrm{C}_{12}\right), 129.12\left(\mathrm{C}_{9}\right.$, $\left.\mathrm{C}_{10}\right), 130.59\left(\mathrm{C}_{5}\right), 132.86\left(\mathrm{C}_{14}\right), 136.44\left(\mathrm{C}_{13}\right), 144.95\left(\mathrm{C}_{8}\right), 145.36\left(\mathrm{C}_{1}\right), 149.34\left(\mathrm{C}_{18}\right), 149.43\left(\mathrm{C}_{17}\right)$, $151.76\left(\mathrm{C}_{4}\right), 189.21\left(\mathrm{C}_{7}\right)$. ESI-MS: $m / z 335.1277[\mathrm{M}+\mathrm{H}]^{+}$, Calc. for $\mathrm{C}_{21} \mathrm{H}_{18} \mathrm{O}_{4}: 335.1283$.

4.1.3.6. (2E)-1-\{4'-nitro-[1,1'-biphenyl]-4-yl\}-3-(furan-2-yl)prop-2-en-1-one (7f): Yield 36\%, beige solid; m.p. $183-185^{\circ} \mathrm{C}$; IR $\left(\mathrm{cm}^{-1}\right)$ : v $v_{\max } 1651(\mathrm{C}=\mathrm{O}), 1593(\mathrm{C}=\mathrm{C}), 1514\left(\mathrm{C}=\mathrm{C}_{\mathrm{Ar}}\right), 1512$ y $1334\left(-\mathrm{NO}_{2}\right)$, 1006 (-C-O-C- furane), 817 y $738\left(\mathrm{C}-\mathrm{H}_{\mathrm{Ar}}\right) .{ }^{1} \mathrm{H}-\mathrm{NMR}\left(\mathrm{CDCl}_{3}, 300 \mathrm{MHz}\right): \delta 6.54\left(\mathrm{H}_{2}, \mathrm{dd}, J 1=1.78\right.$ y $J 2$ $=3.45 \mathrm{~Hz}), 6.76\left(\mathrm{H}_{3}, \mathrm{~d}, J=3.45 \mathrm{~Hz}\right), 7.49\left(\mathrm{H}_{6}, \mathrm{~d}, J=15.30 \mathrm{~Hz}\right), 7.56\left(\mathrm{H}_{1}, \mathrm{~d}, J=1.78 \mathrm{~Hz}\right), 7.64\left(\mathrm{H}_{5}, \mathrm{~d}\right.$, $J=15.30 \mathrm{~Hz}), 7.75\left(\mathrm{H}_{15}, \mathrm{H}_{19}, \mathrm{~d}, J=8.60 \mathrm{~Hz}\right), 7.80\left(\mathrm{H}_{11}, \mathrm{H}_{12}, \mathrm{~d}, J=8.90 \mathrm{~Hz}\right), 8.15\left(\mathrm{H}_{16}, \mathrm{H}_{18}, \mathrm{~d}, J=8.60\right.$ $\mathrm{Hz}), 8.34\left(\mathrm{H}_{9}, \mathrm{H}_{10}, \mathrm{~d}, J=8.90 \mathrm{~Hz}\right) ;{ }^{13} \mathrm{C}-\mathrm{NMR}\left(\mathrm{CDCl}_{3}, 75\right.$,Hz): $\delta 112.87\left(\mathrm{C}_{2}\right), 116.81\left(\mathrm{C}_{3}\right), 118.91\left(\mathrm{C}_{6}\right)$, $124.28\left(\mathrm{C}_{16}, \mathrm{C}_{18}\right), 127.69\left(\mathrm{C}_{11}, \mathrm{C}_{12}\right), 128.14\left(\mathrm{C}_{15}, \mathrm{C}_{19}\right), 129.29\left(\mathrm{C}_{9}, \mathrm{C}_{10}\right), 131.12\left(\mathrm{C}_{5}\right), 138.23\left(\mathrm{C}_{8}\right)$, $142.80\left(\mathrm{C}_{13}\right), 145.20\left(\mathrm{C}_{1}\right), 146.37\left(\mathrm{C}_{14}\right), 147.62\left(\mathrm{C}_{17}\right), 151.61\left(\mathrm{C}_{4}\right), 189.04\left(\mathrm{C}_{7}\right)$. ESI-MS: $m / z 320.0926$ $[\mathrm{M}+\mathrm{H}]^{+}$, Calc. for $\mathrm{C}_{21} \mathrm{H}_{18} \mathrm{O}_{4}: 320.0923$. 
4.1.3.7. (2E)-1-\{4'-fluoro-[1,1'-biphenyl]-4-yl\}-3-(furan-2-yl)prop-2-en-1-one (7g): Yield 38\%; beige solid; descomp. p $260{ }^{\circ} \mathrm{C}$; IR $\left(\mathrm{cm}^{-1}\right)$ : $v_{\max } 1654(\mathrm{C}=\mathrm{O}), 1598(\mathrm{C}=\mathrm{C}), 1550\left(\mathrm{C}=\mathrm{C}_{\mathrm{Ar}}\right), 1058$ (-C-O-Cfurane), $813\left(\mathrm{C}-\mathrm{H}_{\mathrm{Ar}}\right) .{ }^{1} \mathrm{H}-\mathrm{NMR}\left(\mathrm{CDCl}_{3}, 300 \mathrm{MHz}\right): \delta 6.53\left(\mathrm{H}_{2}, \mathrm{dd}, J 1=1.8 \mathrm{y} J 2=3.36 \mathrm{~Hz}\right), 6.74\left(\mathrm{H}_{3}, \mathrm{~d}\right.$, $J=3.36 \mathrm{~Hz}), 7.10-7.22\left(\mathrm{H}_{16}, \mathrm{H}_{18}, \mathrm{~m}\right), 7.50\left(\mathrm{H}_{6}, \mathrm{~d}, J=15.42 \mathrm{~Hz}\right), 7.54\left(\mathrm{H}_{1}, \mathrm{~s}_{\text {apparent }}\right), 7.57-7.64\left(\mathrm{H}_{5}, \mathrm{H}_{15}\right.$, $\left.\mathrm{H}_{19}, \mathrm{~m}\right), 7.67\left(\mathrm{H}_{11}, \mathrm{H}_{12}, \mathrm{~d}, J=8.28 \mathrm{~Hz}\right), 8.11\left(\mathrm{H}_{9}, \mathrm{H}_{10}, \mathrm{~d}, J=8.28 \mathrm{~Hz}\right) ;{ }^{13} \mathrm{C}-\mathrm{NMR}\left(\mathrm{CDCl}_{3}, 75 \mathrm{MHz}\right): \delta$ $112.79\left(\mathrm{C}_{2}\right), 115.81\left(\mathrm{C}_{16}\right), 116.09\left(\mathrm{C}_{18}\right), 116.46\left(\mathrm{C}_{3}\right), 119.15\left(\mathrm{C}_{6}\right), 127.16\left(\mathrm{C}_{11}, \mathrm{C}_{12}\right), 128.93\left(\mathrm{C}_{15}\right)$, $129.04\left(\mathrm{C}_{19}\right), 129.15\left(\mathrm{C}_{9}, \mathrm{C}_{10}\right), 130.73\left(\mathrm{C}_{5}\right), 136.09,136.13\left(\mathrm{C}_{14}\right), 136.86\left(\mathrm{C}_{8}\right), 144.48\left(\mathrm{C}_{13}\right), 145.03$ $\left(\mathrm{C}_{1}\right), 151.72\left(\mathrm{C}_{4}\right), 161.36,164.65\left(\mathrm{C}_{17}\right), 189.20\left(\mathrm{C}_{7}\right)$. ESI-MS: $m / z 293.0972[\mathrm{M}+\mathrm{H}]^{+}$, Calc. for $\mathrm{C}_{21} \mathrm{H}_{18} \mathrm{O}_{4}$ : 293.0978 .

4.1.3.8. (2E)-1-([1,1'-biphenyl]-4-yl)-3-(furan-2-yl)prop-2-en-1-one (7h): Yield 65\%; yellow solid; m.p. $143-145^{\circ} \mathrm{C}$; IR $\left(\mathrm{cm}^{-1}\right)$ : v $v_{\max } 1654(\mathrm{C}=\mathrm{O}), 1593(\mathrm{C}=\mathrm{C}), 1544\left(\mathrm{C}=\mathrm{C}_{\mathrm{Ar}}\right), 1012$ (-C-O-C- furane), 736 $\left(\mathrm{C}-\mathrm{H}_{\mathrm{Ar}}\right) .{ }^{1} \mathrm{H} \mathrm{NMR}\left(\mathrm{CDCl}_{3}, 300 \mathrm{MHz}\right): \delta 6.33\left(\mathrm{H}_{2}, \mathrm{~s}_{\text {apparent }}\right), 6.74\left(\mathrm{H}_{3}, \mathrm{~s}_{\text {apparent }}\right), 7.36-7.63\left(\mathrm{H}_{1}, \mathrm{H}_{5}, \mathrm{H}_{6}\right.$, $\left.\mathrm{H}_{15}, \mathrm{H}_{17}, \mathrm{H}_{19}, \mathrm{~m}\right), 7.66\left(\mathrm{H}_{16}, \mathrm{H}_{18}, \mathrm{~d}, J=7.2 \mathrm{~Hz}\right), 7.73\left(\mathrm{H}_{11}, \mathrm{H}_{12}, \mathrm{~d}, J=8.0 \mathrm{~Hz}\right), 8.12\left(\mathrm{H}_{9}, \mathrm{H}_{10}, \mathrm{~d}, J=8.0\right.$ $\mathrm{Hz} ;{ }^{13} \mathrm{C} \mathrm{NMR}\left(\mathrm{CDCl}_{3}, 75 \mathrm{MHz}\right) \delta 189.38(\mathrm{C}=\mathrm{O}), 151.85\left(\mathrm{C}_{4}\right), 145.63\left(\mathrm{C}_{1}\right), 145.07\left(\mathrm{C}_{13}\right), 140.10\left(\mathrm{C}_{14}\right)$, $136.98\left(\mathrm{C}_{8}\right), 130.74\left(\mathrm{C}_{5}\right), 129.19\left(\mathrm{C}_{9,10}\right), 129.10\left(\mathrm{C}_{18,16}\right), 128.33\left(\mathrm{C}_{16,18}\right), 127.42\left(\mathrm{C}_{11,12,15,17,19}\right), 119.36$ $\left(\mathrm{C}_{6}\right), 116.44\left(\mathrm{C}_{3}\right), 112.85\left(\mathrm{C}_{2}\right)$; ESI-MS: $\mathrm{m} / \mathrm{z}[\mathrm{M}+\mathrm{H}]^{+}$, Calc. for $\mathrm{C}_{19} \mathrm{H}_{14} \mathrm{O}_{2}: 274,0994$.

4.1.3.9. (2E)-1-(4'-methoxy-[1,1'-biphenyl]-4-yl) -3-(furan-2-yl)prop-2-en-1-one (7i): Yield 85\%; yellow solid; descomp. p. $170{ }^{\circ} \mathrm{C}$; IR $\left(\mathrm{cm}^{-1}\right)$ : $v_{\max } 1654(\mathrm{C}=\mathrm{O}), 1598(\mathrm{C}=\mathrm{C}), 1014$ (-C-O-C- furane), 817 $\left(\mathrm{C}-\mathrm{H}_{\mathrm{Ar}}\right) .{ }^{1} \mathrm{H}$ NMR $\left(\mathrm{CDCl}_{3}, 300 \mathrm{MHz}\right): \delta 4.30\left(-\mathrm{O}-\underline{\mathrm{C}}_{3}, \mathrm{~s}\right), 6.99\left(\mathrm{H}_{2}, \mathrm{~s}_{\text {apparent }}\right), 7.23\left(\mathrm{H}_{3}, \mathrm{~s}_{\text {apparent }}\right), 7.45$ $\left(\mathrm{H}_{15}, \mathrm{H}_{19}, \mathrm{~d}, J=8.3 \mathrm{~Hz}\right), 7.87-8.09\left(\mathrm{H}_{1}, \mathrm{H}_{5}, \mathrm{H}_{6}, \mathrm{H}_{16}, \mathrm{H}_{17}, \mathrm{H}_{18}, \mathrm{~m}\right), 8.14\left(\mathrm{H}_{11}, \mathrm{H}_{12}, \mathrm{~d}, J=8.0 \mathrm{~Hz}\right), 8.51$ $\left(\mathrm{H}_{9}, \mathrm{H}_{10}, \mathrm{~d}, J=8.0 \mathrm{~Hz}\right) ;{ }^{13} \mathrm{C} \mathrm{NMR}\left(\mathrm{CDCl}_{3}, 75 \mathrm{MHz}\right) \delta: 59.15\left(-\mathrm{O}-\mathrm{CH}_{3}\right), 116.71\left(\mathrm{C}_{2}\right), 118.31\left(\mathrm{C}_{11}, \mathrm{C}_{12}\right)$, $120.70\left(\mathrm{C}_{3}\right), 122.95\left(\mathrm{C}_{6}\right), 130.60\left(\mathrm{C}_{9}, \mathrm{C}_{10}\right), 132.27\left(\mathrm{C}_{16}, \mathrm{C}_{18}\right), 133.05\left(\mathrm{C}_{15}, \mathrm{C}_{19}\right), 134.92\left(\mathrm{C}_{5}\right), 136.12$ $\left(\mathrm{C}_{14}\right), 139.92\left(\mathrm{C}_{13}\right), 149.27\left(\mathrm{C}_{8}\right), 149.37\left(\mathrm{C}_{1}\right), 155.50\left(\mathrm{C}_{4}\right), 163.87\left(\mathrm{C}_{17}\right), 194.19\left(\mathrm{C}_{7}\right) ;$ ESI-MS: m/z [M $+\mathrm{H}]^{+}$, Calc. for $\mathrm{C}_{19} \mathrm{H}_{14} \mathrm{O}_{2}: 274,0994$.

4.1.3.10. (2E)-1-\{6-hydroxy-2',3'-dimethoxy-[1,1'-biphenyl]-3-yl\}-3-(furan-2-yl)prop-2-en-1-one (8a) Yield 61\%, beige solid; ${ }^{1} \mathrm{H}-\mathrm{NMR}\left(\mathrm{CDCl}_{3}, 300 \mathrm{MHz}\right): \delta 3.71\left(-\mathrm{O}-\mathrm{C}_{3}, \mathrm{~s}\right), 3.95\left(-\mathrm{O}-\mathrm{C}_{3}, \mathrm{~s}\right), 6.51\left(\mathrm{H}_{2}\right.$, dd, $J 1=1.8$ y $J 2=3.15 \mathrm{~Hz}), 6.71\left(\mathrm{H}_{3}, \mathrm{~d}, J=3.15 \mathrm{~Hz}\right), 7.02\left(\mathrm{H}_{16}, \mathrm{H}_{17}, \mathrm{~d}, J=8.11 \mathrm{~Hz}\right), 7.14\left(\mathrm{H}_{11}, \mathrm{~d}, J=\right.$ $8.50 \mathrm{~Hz}), 7.23\left(\mathrm{H}_{15}, \mathrm{~d}, J=8.11 \mathrm{~Hz}\right), 7.49\left(\mathrm{H}_{1}, \mathrm{H}_{6}, \mathrm{~d}, J=15.32 \mathrm{~Hz}\right), 7.62\left(\mathrm{H}_{5}, \mathrm{~d}, J=15.32 \mathrm{~Hz}\right), 8.05$ $\left(\mathrm{H}_{9}, \mathrm{dd}, J 1=2.10\right.$ y $\left.J 2=8.50 \mathrm{~Hz}\right), 8.11\left(\mathrm{H}_{10}, \mathrm{~d}, J=2.10 \mathrm{~Hz}\right) ;{ }^{13} \mathrm{C}-\mathrm{NMR}\left(\mathrm{CDCl}_{3}, 75 \mathrm{MHz}\right): \delta 56.02(-$ $\left.\mathrm{O}-\underline{\mathrm{CH}}_{3}\right), 61.76\left(-\mathrm{O}-\underline{\mathrm{C}} \mathrm{H}_{3}\right), 112.12\left(\mathrm{C}_{16}\right), 112.66\left(\mathrm{C}_{2}\right), 115.97\left(\mathrm{C}_{3}\right), 118.40\left(\mathrm{C}_{11}\right), 119.18\left(\mathrm{C}_{6}\right), 124.01$ $\left(\mathrm{C}_{17}\right), 125.85\left(\mathrm{C}_{15}\right), 125.93\left(\mathrm{C}_{8}\right), 130.15\left(\mathrm{C}_{5}\right), 130.41\left(\mathrm{C}_{9}\right), 131.46\left(\mathrm{C}_{13}\right), 131.67\left(\mathrm{C}_{14}\right), 132.53\left(\mathrm{C}_{10}\right)$, 
$144.77\left(\mathrm{C}_{1}\right), 145.25\left(\mathrm{C}_{19}\right), 151.85\left(\mathrm{C}_{4}\right), 152.82\left(\mathrm{C}_{18}\right), 158.24\left(\mathrm{C}_{12}\right), 188.16\left(\mathrm{C}_{7}\right)$. ESI-MS: $m / z 351.1225$ $[\mathrm{M}+\mathrm{H}]^{+}$, Calc. for $\mathrm{C}_{21} \mathrm{H}_{18} \mathrm{O}_{5}: 351.1232$.

4.1.3.11. (2E)-1-\{6-hydroxy-2',4'-dimethoxy-[1,1'-biphenyl]-3-yl\}-3-(furan-2-yl)prop-2-en-1-one (8b) Yield 45\%; yellow oil; ${ }^{1} \mathrm{H}-\mathrm{NMR}\left(\mathrm{CDCl}_{3}, 300 \mathrm{MHz}\right): \delta 3.88\left(-\mathrm{O}-\mathrm{CH}_{3}, \mathrm{~s}\right), 6.50\left(\mathrm{H}_{2}, \mathrm{dd}, J 1=1.5\right.$ y $J 2=$ $3.27 \mathrm{~Hz}), 6.62\left(\mathrm{H}_{3}, \mathrm{~d}, J=3.27 \mathrm{~Hz}\right), 6.68\left(\mathrm{H}_{16}, \mathrm{H}_{18}, \mathrm{dd}, J 1=2.3\right.$ y $\left.J 2=8.5 \mathrm{~Hz}\right), 7.08\left(\mathrm{H}_{11}, \mathrm{~d}, J=8.50\right.$ $\mathrm{Hz}), 7.30\left(\mathrm{H}_{15}, \mathrm{~d}, J=8.50 \mathrm{~Hz}\right), 7.48\left(\mathrm{H}_{1}, \mathrm{H}_{5}, \mathrm{~d}, J=15.27 \mathrm{~Hz}\right), 7.59\left(\mathrm{H}_{6}, \mathrm{~d}, J=15.27 \mathrm{~Hz}\right), 7.97\left(\mathrm{H}_{10}, \mathrm{~d}\right.$, $J=2.10 \mathrm{~Hz}), 8.01\left(\mathrm{H}_{9}, \mathrm{dd}, J 1=2.10\right.$ y $\left.J 2=8.50 \mathrm{~Hz}\right) ;{ }^{13} \mathrm{C}-\mathrm{NMR}\left(\mathrm{CDCl}_{3}, 75 \mathrm{MHz}\right): \delta 55.62\left(-\mathrm{O}-\underline{\mathrm{CH}}_{3}\right)$, $56.21\left(-\mathrm{O}-\underline{\mathrm{CH}}_{3}\right), 99.24\left(\mathrm{C}_{18}\right), 106.33\left(\mathrm{C}_{16}\right), 112.63\left(\mathrm{C}_{2}\right), 115.85\left(\mathrm{C}_{11}\right), 117.08\left(\mathrm{C}_{3}\right), 118.13\left(\mathrm{C}_{6}\right), 119.30$ $\left(\mathrm{C}_{13}\right), 125.98\left(\mathrm{C}_{10}\right), 129.96\left(\mathrm{C}_{15}\right), 130.01\left(\mathrm{C}_{9}\right), 131.33\left(\mathrm{C}_{5}\right), 132.59\left(\mathrm{C}_{14}\right), 133.18\left(\mathrm{C}_{8}\right), 144.70\left(\mathrm{C}_{1}\right)$, $151.88\left(\mathrm{C}_{4}\right), 156.73\left(\mathrm{C}_{12}\right), 158.10\left(\mathrm{C}_{19}\right), 161.40\left(\mathrm{C}_{17}\right), 188.23\left(\mathrm{C}_{7}\right)$. ESI-MS: $m / z 351.1235[\mathrm{M}+\mathrm{H}]^{+}$, Calc. for $\mathrm{C}_{21} \mathrm{H}_{18} \mathrm{O}_{5}: 351.1232$.

4.1.3.12. (2E)-1-\{6-hydroxy-2',5'-dimethoxy-[1,1'-biphenyl]-3-yl\}-3-(furan-2-yl)prop-2-en-1-one (8c) Yield 65\%, yellow oil; ${ }^{1} \mathrm{H}-\mathrm{NMR}\left(\mathrm{CDCl}_{3}, 600 \mathrm{MHz}\right): \delta 3.83\left(-\mathrm{O}-\underline{\mathrm{C}}_{3}, \mathrm{~s}\right), 3.85\left(-\mathrm{O}-\underline{\mathrm{H}}_{3}, \mathrm{~s}\right), 6.51\left(\mathrm{H}_{2}\right.$, $\mathrm{dd}, J 1=1.8$ y $J 2=3.47 \mathrm{~Hz}), 6.70\left(\mathrm{H}_{3}, \mathrm{~d}, J=3.47 \mathrm{~Hz}\right), 6.94\left(\mathrm{H}_{15}, \mathrm{~d}, J=3.05 \mathrm{~Hz}\right), 6.97\left(\mathrm{H}_{17}, \mathrm{dd}, J 1=\right.$ 3.05 y $J 2=8.90 \mathrm{~Hz}), 7.01\left(\mathrm{H}_{11}, \mathrm{~d}, J=8.90 \mathrm{~Hz}\right), 7.11\left(\mathrm{H}_{18}, \mathrm{~d}, J=8.90 \mathrm{~Hz}\right), 7.49\left(\mathrm{H}_{5}, \mathrm{~d}, J=15.30 \mathrm{~Hz}\right)$, $7.51\left(\mathrm{H}_{1}, \mathrm{~d}, J=1.80 \mathrm{~Hz}\right), 7.60\left(\mathrm{H}_{6}, \mathrm{~d}, J=15.30 \mathrm{~Hz}\right), 8.05\left(\mathrm{H}_{9}, \mathrm{H}_{10}\right.$, dd, $J 1=2.25$ y $\left.J 2=6.90 \mathrm{~Hz}\right) ;{ }^{13} \mathrm{C}-$ NMR (CDCl $3,150 \mathrm{MHz}): \delta 55.91\left(-\mathrm{O}-\underline{\mathrm{CH}}_{3}\right), 57.22\left(-\mathrm{O}-\underline{\mathrm{CH}}_{3}\right), 112.66\left(\mathrm{C}_{18}\right), 113.40\left(\mathrm{C}_{2}\right), 114.90\left(\mathrm{C}_{17}\right)$, $116.01\left(\mathrm{C}_{11}\right), 117.73\left(\mathrm{C}_{15}\right), 117.74\left(\mathrm{C}_{3}\right), 119.14\left(\mathrm{C}_{6}\right), 126.27\left(\mathrm{C}_{14}\right), 127.10\left(\mathrm{C}_{13}\right), 130.15\left(\mathrm{C}_{10}\right), 130.35$ $\left(\mathrm{C}_{9}\right), 131.44\left(\mathrm{C}_{5}\right), 132.46\left(\mathrm{C}_{8}\right), 144.77\left(\mathrm{C}_{1}\right), 149.61\left(\mathrm{C}_{4}\right), 151.80\left(\mathrm{C}_{12}\right), 154.82\left(\mathrm{C}_{19}\right), 158.16\left(\mathrm{C}_{16}\right)$, 188.16 $\left(\mathrm{C}_{7}\right)$. ESI-MS: $m / z$ 351.1232 $[\mathrm{M}+\mathrm{H}]^{+}$, Calc. for $\mathrm{C}_{21} \mathrm{H}_{18} \mathrm{O}_{5}: 351.1232$.

4.1.3.13. (2E)-1-\{6-hydroxy-3',4'-dimethoxy-[1,1'-biphenyl]-3-yl\}-3-(furan-2-yl)prop-2-en-1-one (8e) Yield 64\%, yellow solid; m.p. 145-147 ${ }^{\circ} \mathrm{C}$; IR $\left(\mathrm{cm}^{-1}\right): v_{\max } 3116(-\mathrm{OH}), 2995\left(-\mathrm{CH}_{3}\right), 1645(\mathrm{C}=\mathrm{O})$, $1600(\mathrm{C}=\mathrm{C}), 1556\left(\mathrm{C}=\mathrm{C}_{\mathrm{Ar}}\right), 1271$ y $1049\left(-\mathrm{OCH}_{3} \mathrm{Ar}\right), 1022(-\mathrm{C}-\mathrm{O}-\mathrm{C}-$ furane $), 813$ y $763\left(\mathrm{C}-\mathrm{H}_{\mathrm{Ar}}\right) .{ }^{1} \mathrm{H}-$ NMR $\left(\mathrm{CDCl}_{3}, 600 \mathrm{MHz}\right): \delta 3.92\left(-\mathrm{O}-\mathrm{CH}_{3}, \mathrm{~s}\right), 3.94\left(-\mathrm{O}-\mathrm{CH}_{3}, \mathrm{~s}\right), 6.51\left(\mathrm{H}_{2}, \mathrm{dd}, J 1=1.5\right.$ y $\left.J 2=3.44 \mathrm{~Hz}\right)$, $6.70\left(\mathrm{H}_{3}, \mathrm{~d}, J=3.44 \mathrm{~Hz}\right), 6.98\left(\mathrm{H}_{19}, \mathrm{~d}, J=2.02 \mathrm{~Hz}\right), 6.99\left(\mathrm{H}_{16}, \mathrm{~d}, J=8.20 \mathrm{~Hz}\right), 7.04\left(\mathrm{H}_{9}, \mathrm{dd}, J 1=2.02 \mathrm{y}\right.$ $J 2=7.45 \mathrm{~Hz}), 7.06-7.10\left(\mathrm{H}_{15}, \mathrm{~m}\right), 7.48\left(\mathrm{H}_{5}, \mathrm{~d}, J=15.30 \mathrm{~Hz}\right), 7.51\left(\mathrm{H}_{1}, \mathrm{~d}, J=1.50 \mathrm{~Hz}\right), 7.60\left(\mathrm{H}_{6}, \mathrm{~d}, J=\right.$ $15.30 \mathrm{~Hz}), 7.98-8.01\left(\mathrm{H}_{10}, \mathrm{H}_{11}, \mathrm{~m}\right) ;{ }^{13} \mathrm{C}-\mathrm{NMR}\left(\mathrm{CDCl}_{3}, 150 \mathrm{MHz}\right): \delta 56.03\left(-\mathrm{O}-\underline{\mathrm{CH}}_{3}\right), 56.07\left(-\mathrm{O}-\underline{\mathrm{CH}}_{3}\right)$, $111.72\left(\mathrm{C}_{19}\right), 112.20\left(\mathrm{C}_{16}\right), 112.70\left(\mathrm{C}_{2}\right), 115.85\left(\mathrm{C}_{11}\right), 116.15\left(\mathrm{C}_{3}\right), 119.01\left(\mathrm{C}_{6}\right), 121.24\left(\mathrm{C}_{13}\right), 128.24$ $\left(\mathrm{C}_{15}\right), 128.38\left(\mathrm{C}_{10}\right), 130.06\left(\mathrm{C}_{5}\right), 130.24\left(\mathrm{C}_{9}\right), 131.12\left(\mathrm{C}_{14}\right), 131.25\left(\mathrm{C}_{8}\right), 144.83\left(\mathrm{C}_{1}\right), 149.15\left(\mathrm{C}_{18}\right)$, $149.70\left(\mathrm{C}_{17}\right), 151.77\left(\mathrm{C}_{4}\right), 157.07\left(\mathrm{C}_{12}\right), 188.24\left(\mathrm{C}_{7}\right)$. ESI-MS: $m / z 351.1230[\mathrm{M}+\mathrm{H}]^{+}$, Calc. for $\mathrm{C}_{21} \mathrm{H}_{18} \mathrm{O}_{5}$ : 351.1232 . 
4.1.3.14. (2E)-1-\{6-hydroxy-4'-nitro-[1,1'-biphenyl]-3-yl\}-3-(furan-2-yl)prop-2-en-1-one (8f)

Yield 31\%, yellow solid; m.p. 154-157 ${ }^{\circ} \mathrm{C}$; IR $\left(\mathrm{cm}^{-1}\right)$ : $v_{\max } 3111(-\mathrm{OH}), 1643(\mathrm{C}=\mathrm{O}), 1602(\mathrm{C}=\mathrm{C})$, $1562\left(\mathrm{C}=\mathrm{C}_{\mathrm{Ar}}\right), 1510$ y $1346\left(-\mathrm{NO}_{2}\right), 1014$ (-C-O-C- furane), $850\left(\mathrm{C}-\mathrm{H}_{\mathrm{Ar}}\right) .{ }^{1} \mathrm{H}-\mathrm{NMR}\left(\mathrm{CDCl}_{3}, 300 \mathrm{MHz}\right): \delta$ $6.59\left(\mathrm{H}_{2}, \mathrm{~s}_{\text {apparente }}\right), 6.88\left(\mathrm{H}_{3}, \mathrm{~d}, J=3.50 \mathrm{~Hz}\right), 7.06\left(\mathrm{H}_{9}, \mathrm{dd}, J 1=5.76\right.$ y $\left.J 2=8.45 \mathrm{~Hz}\right), 7.55-7.60\left(\mathrm{H}_{15}\right.$, $\left.\mathrm{H}_{19}, \mathrm{~m}\right), 7.68\left(\mathrm{H}_{1}, \mathrm{~s}\right), 7.84-7.90\left(\mathrm{H}_{5}, \mathrm{H}_{6}, \mathrm{~m}\right), 7.99-8.05\left(\mathrm{H}_{11}, \mathrm{~m}\right), 8.08\left(\mathrm{H}_{10}, \mathrm{~d}, J=5.76 \mathrm{~Hz}\right), 8.25-8.33$ $\left(\mathrm{H}_{16}, \mathrm{H}_{18}, \mathrm{~m}\right) ;{ }^{13} \mathrm{C}-\mathrm{NMR}\left(\mathrm{CDCl}_{3}, 75 \mathrm{MHz}\right): \delta 112.43\left(\mathrm{C}_{2}\right), 115.86\left(\mathrm{C}_{11}\right), 116.08\left(\mathrm{C}_{3}\right), 118.39\left(\mathrm{C}_{6}\right)$, $122.80\left(\mathrm{C}_{16}, \mathrm{C}_{18}\right), 126.45\left(\mathrm{C}_{13}\right), 129.98\left(\mathrm{C}_{9}\right), 130.06\left(\mathrm{C}_{15}, \mathrm{C}_{19}\right), 130.17\left(\mathrm{C}_{10}\right), 130.82\left(\mathrm{C}_{5}\right), 131.44\left(\mathrm{C}_{14}\right)$, $144.74\left(\mathrm{C}_{8}\right), 145.37\left(\mathrm{C}_{1}\right), 146.78\left(\mathrm{C}_{17}\right), 151.73\left(\mathrm{C}_{4}\right), 159.55\left(\mathrm{C}_{12}\right), 188.44\left(\mathrm{C}_{7}\right)$. ESI-MS: $m / z 336.0872$ $[\mathrm{M}+\mathrm{H}]^{+}$, Calc. for $\mathrm{C}_{21} \mathrm{H}_{18} \mathrm{O}_{5}: 336.0872$.

4.1.3.15. (2E)-1-\{4'-fluoro-6-hydroxy-[1,1'-biphenyl]-3-yl\}-3-(furan-2-yl)prop-2-en-1-one (8g)

Yield 54\%, yellow solid; m.p. 164-166 ${ }^{\circ} \mathrm{C}$; IR $\left(\mathrm{cm}^{-1}\right): v_{\max } 3149(-\mathrm{OH}), 1649(\mathrm{C}=\mathrm{O}), 1600(\mathrm{C}=\mathrm{C})$, $1571\left(\mathrm{C}=\mathrm{C}_{\mathrm{Ar}}\right), 1006$ (-C-O-C- furane), 813 y $744\left(\mathrm{C}-\mathrm{H}_{\mathrm{Ar}}\right) .{ }^{1} \mathrm{H}-\mathrm{NMR}\left(\mathrm{CDCl}_{3}, 300 \mathrm{MHz}\right): \delta 6.48\left(\mathrm{H}_{2}, \mathrm{dd}\right.$, $J 1=1.70$ у $J 2=3.20 \mathrm{~Hz}), 6.68\left(\mathrm{H}_{3}, \mathrm{~d}, J=3.20 \mathrm{~Hz}\right), 6.97\left(\mathrm{H}_{11}, \mathrm{~d}, J=8.42 \mathrm{~Hz}\right), 7.05-7.17\left(\mathrm{H}_{16}, \mathrm{H}_{18}\right.$, m), $7.44\left(\mathrm{H}_{5}, \mathrm{~d}, J=15.31 \mathrm{~Hz}\right), 7.48-7.54\left(\mathrm{H}_{1}, \mathrm{H}_{6}, \mathrm{H}_{19}, \mathrm{~m}\right), 7.57\left(\mathrm{H}_{15}, \mathrm{~d}, J=8.08 \mathrm{~Hz}\right), 7.90\left(\mathrm{H}_{9}, \mathrm{dd}, J 1=\right.$ 2.10 y $J 2=8.42 \mathrm{~Hz}), 7.95\left(\mathrm{H}_{10}, \mathrm{~d}, J=2.10 \mathrm{~Hz}\right) ;{ }^{13} \mathrm{C}-\mathrm{NMR}\left(\mathrm{CDCl}_{3}, 75 \mathrm{MHz}\right): \delta 112.68\left(\mathrm{C}_{2}\right), 115.16$ $\left(\mathrm{C}_{11}\right), 115.44\left(\mathrm{C}_{16}\right), 115.97\left(\mathrm{C}_{18}\right), 116.18\left(\mathrm{C}_{3}\right), 119.02\left(\mathrm{C}_{6}\right), 127.76\left(\mathrm{C}_{13}\right), 129.94\left(\mathrm{C}_{9}\right), 130.25\left(\mathrm{C}_{15}\right)$, $130.98\left(\mathrm{C}_{19}\right), 131.09\left(\mathrm{C}_{10}\right), 131.87\left(\mathrm{C}_{5}\right), 133.39\left(\mathrm{C}_{8}\right), 133.44\left(\mathrm{C}_{14}\right), 144.87\left(\mathrm{C}_{1}\right), 151.73\left(\mathrm{C}_{4}\right), 158.54$ $\left(\mathrm{C}_{12}\right), 160.63,163.90\left(\mathrm{C}_{17}\right), 188.67\left(\mathrm{C}_{7}\right)$. ESI-MS: $m / z 309.0923[\mathrm{M}+\mathrm{H}]^{+}$, Calcd for $\mathrm{C}_{21} \mathrm{H}_{18} \mathrm{O}_{5}$ : 309.0927 . 
4.1.3.16. (2E)-1-(6-hydroxy-[1,1'-biphenyl]-3-yl)-3-(furan-2-yl)prop-2-en-1-one (8h): Yield 66\%; orange solid; m.p. $115-118^{\circ} \mathrm{C}$; IR $\left(\mathrm{cm}^{-1}\right)$ : $v_{\max } 3429(-\mathrm{OH}), 1710(\mathrm{C}=\mathrm{O}), 1647(\mathrm{C}=\mathrm{C}), 1600\left(\mathrm{C}=\mathrm{C}_{\mathrm{Ar}}\right)$, 1006 (-C-O-C- furane), 813 y $744\left(\mathrm{C}-\mathrm{H}_{\mathrm{Ar}}\right) .{ }^{1} \mathrm{H}-\mathrm{NMR}\left(\mathrm{CDCl}_{3}, 300 \mathrm{MHz}\right): \delta 6.51\left(\mathrm{H}_{2}, \mathrm{dd}, J 1=3.4, J 2=\right.$ $1.8 \mathrm{~Hz}), 6.70\left(\mathrm{H}_{3}, \mathrm{~d}, J=3.4 \mathrm{~Hz}\right), 7.13-7.05\left(\mathrm{H}_{11}, \mathrm{~m}\right), 7.56-7.39\left(\mathrm{H}_{16}, \mathrm{H}_{18}, \mathrm{H}_{5}, \mathrm{H}_{1}, \mathrm{H}_{19}, \mathrm{H}_{15}, \mathrm{H}_{9}, \mathrm{~m}\right)$, $7.61\left(\mathrm{H}_{6}, \mathrm{~d}, J=15.3 \mathrm{~Hz}\right), 8.09-7.96\left(\mathrm{H}_{10}, \mathrm{H}_{17}, \mathrm{~m}\right) ;{ }^{13} \mathrm{C} \mathrm{NMR}\left(\mathrm{CDCl}_{3}, 75 \mathrm{MHz}\right): \delta 112.71\left(\mathrm{C}_{2}\right), 116.16$ $\left(\mathrm{C}_{16}, \mathrm{C}_{18}\right), 119.07\left(\mathrm{C}_{3}\right), 128.33\left(\mathrm{C}_{6}\right), 128.45\left(\mathrm{C}_{17}\right), 129.21\left(\mathrm{C}_{9}, \mathrm{C}_{10}\right), 129.36\left(\mathrm{C}_{15}, \mathrm{C}_{19}\right), 130.26\left(\mathrm{C}_{11}\right)$, $130.34\left(\mathrm{C}_{5}\right), 131.16\left(\mathrm{C}_{13}\right), 131.56\left(\mathrm{C}_{8}\right), 136,27\left(\mathrm{C}_{14}\right), 144.86\left(\mathrm{C}_{1}\right), 151.79\left(\mathrm{C}_{4}\right), 157.19\left(\mathrm{C}_{12}\right), 188.39$ $\left(\mathrm{C}_{7}\right)$; ESI-MS: $m / z[\mathrm{M}+\mathrm{H}]^{+}$, Calcd for $\mathrm{C}_{19} \mathrm{H}_{14} \mathrm{O}_{3}: 290.0943$.

4.1.3.17. (2E)-1-(6-hydroxy-4'-methoxy-[1,1'-biphenyl]-3-yl)-3-(furan-2-yl)prop-2-en-1-one (8i): Yield 74\%; orange solid; m.p. 144-146 ${ }^{\circ} \mathrm{C}$; IR $\left(\mathrm{cm}^{-1}\right): v_{\max } 3427(-\mathrm{OH}), 1653(\mathrm{C}=\mathrm{O}), 1602(\mathrm{C}=\mathrm{C})$, 1016 (-C-O-C- furane). ${ }^{1} \mathrm{H}$ NMR $\left(\mathrm{CDCl}_{3}, 300 \mathrm{MHz}\right): \delta 3.86\left(-\mathrm{O}-\mathrm{CH}_{3}, \mathrm{~s}\right), 6.21(-\mathrm{OH}, \mathrm{s}), 6.51\left(\mathrm{H}_{2}, \mathrm{dd}, J=\right.$ 3.4, $1.8 \mathrm{~Hz}), 6.70\left(\mathrm{H}_{3}, \mathrm{~d}, J=3.4 \mathrm{~Hz}\right), 6.99-7.10\left(\mathrm{H}_{11}, \mathrm{H}_{16}, \mathrm{H}_{18}, \mathrm{~m}\right), 7.43\left(\mathrm{H}_{15}, \mathrm{H}_{19}, \mathrm{~d}, J=8.82 \mathrm{~Hz}\right)$, $7.48-7.54\left(\mathrm{H}_{1}, \mathrm{H}_{5}, \mathrm{~m}\right), 7.60\left(\mathrm{H}_{6}, \mathrm{~d}, J=15.3 \mathrm{~Hz}\right), 8.04-7.94\left(\mathrm{H}_{9}, \mathrm{H}_{10}, \mathrm{~m}\right) ;{ }^{13} \mathrm{C} \mathrm{NMR}\left(\mathrm{CDCl}_{3}, 75\right.$ $\mathrm{MHz}): \delta 55.44\left(-\mathrm{O}-\underline{\mathrm{CH}}_{3}\right), 112.69\left(\mathrm{C}_{2}\right), 114.84\left(\mathrm{C}_{15}, \mathrm{C}_{19}\right), 115.94\left(\mathrm{C}_{3}\right), 116.08\left(\mathrm{C}_{11}\right), 119.12\left(\mathrm{C}_{6}\right), 128.14$ $\left(\mathrm{C}_{9}\right), 128.22\left(\mathrm{C}_{10}\right), 129.96\left(\mathrm{C}_{5}\right), 130.26\left(\mathrm{C}_{14}\right), 130.40\left(\mathrm{C}_{16}, \mathrm{C}_{18}\right), 131.16\left(\mathrm{C}_{13}\right), 131.46\left(\mathrm{C}_{8}\right), 144.82\left(\mathrm{C}_{1}\right)$, $151.81\left(\mathrm{C}_{4}\right), 157.18\left(\mathrm{C}_{17}\right), 159.67\left(\mathrm{C}_{12}\right), 188.38\left(\mathrm{C}_{7}\right)$; ESI-MS: $m / z[\mathrm{M}+\mathrm{H}]^{+}$, Calcd for $\mathrm{C}_{20} \mathrm{H}_{16} \mathrm{O}_{4}$ : 320.1049 


\subsection{Biological activity assays}

The compounds were subjected to in vitro evaluation as regards their cytotoxicity and antitrypanosomal activity against U-937 human cells and intracellular amastigotes of $T$. cruzi, respectively.

\subsubsection{In vitro Cytotoxicity}

The cytotoxic activity of the compounds was assessed based on the viability of the human promonocytic cell line U-937 (ATCC CRL-1593.2 ${ }^{\mathrm{TM}}$ ) evaluated by the MTT (3-(4,5-dimethylthiazol2-yl)-2,5-diphenyltetrazolium bromide) assay following the methodology described previously [23]. Briefly, cells grown in tissue flasks were harvested and washed with phosphate buffered saline (PBS) by centrifuging. Cells were counted and adjusted at $1 \times 10^{6}$ cells $/ \mathrm{mL}$ of RPMI-1640 supplemented with complete $10 \%$ Fetal Bovine Serum (FBS) and $1 \%$ antibiotics $(100 \mathrm{U} / \mathrm{mL}$ penicillin and $0.1 \mathrm{mg} / \mathrm{mL}$ streptomycin). One hundred $\mu \mathrm{L}$ were dispensed into each well of a 96-well cell-culture plate and then $100 \mathrm{~mL}$ of RPMI-1640 and the corresponding concentrations of the compounds were added, starting at $200 \mu \mathrm{g} / \mathrm{mL}$ in duplicate. Plates were incubated at $37{ }^{\circ} \mathrm{C}, 5 \% \mathrm{CO}_{2}$ during $72 \mathrm{~h}$ in the presence of extracts. The effect of compounds was determined by measuring the activity of the mitochondrial dehydrogenase by adding $10 \mu \mathrm{L} /$ well of MTT solution $(0.5 \mathrm{mg} / \mathrm{mL})$ and incubation at $37{ }^{\circ} \mathrm{C}$ for $3 \mathrm{~h}$. The reaction was stopped by adding $100 \mu \mathrm{L} /$ well of $50 \%$ isopropanol solution with $10 \%$ sodium dodecyl sulfate and 30 min incubation. Cell viability was determined based on the quantity of formazan produced according to the intensity of color (absorbance) registered as optical densities (O.D) obtained at $570 \mathrm{~nm}$ in a spectrophotometer (Varioskan ${ }^{\mathrm{TM}}$ Flash Multimode Reader - Thermo Scientific, USA). Cells cultured in absence of compounds were used as control of viability (negative control), while meglumine antimoniate (Sbv) and amphotericin B (AmB) were used as control for cytotoxicity (noncytotoxic and cytotoxic drugs, respectively). Assays were conducted in two independent runs with three replicates per each concentration tested. 


\subsubsection{In vitro Trypanocidal Activity}

Compounds were tested on intracellular amastigotes of $T$. cruzi, Tulahuen strain transfected with $\beta$ galactosidase gene (donated by Dr. F. S. Buckner, University of Washington) [25]. The activity was determined according to the ability of the compound to reduce the infection of U-937 cells by $T$. cruzi as described elsewhere [38]. Following the procedure described above, anti-T. cruzi activity was initially screened at a single concentration of $20 \mathrm{mg} / \mathrm{mL}$. In this case, $100 \mu \mathrm{L}$ of U-937 human cells at a concentration of $2.5 \times 10^{5}$ cells $/ \mathrm{mL}$ in RPMI-1640, 10\% SFB and $0.1 \mu \mathrm{g} / \mathrm{mL}$ of PMA were placed in each well of 96-well plates and then infected with phase growth epimastigotes in 5:1 (parasites per cell) ratio and incubated at $34{ }^{\circ} \mathrm{C}, 5 \% \mathrm{CO}_{2}$. After 24 hours of incubation, $20 \mu \mathrm{g} / \mathrm{mL}$ of each compound were added to infected cells. After $72 \mathrm{~h}$ of incubation, the effect of all compounds on viability of intracellular amastigotes was determined by measuring the $\beta$-galactosidase activity by spectrophotometry adding $100 \mu \mathrm{M} \mathrm{CPRG}$ and $0.1 \%$ nonidet P-40 to each well. After $3 \mathrm{~h}$ of incubation, plates were read at $570 \mathrm{~nm}$ in a spectrophotometer (Varioskan ${ }^{\mathrm{TM}}$ Flash Multimode Reader - Thermo Scientific, USA) and intensity of color (absorbance) was registered as O.D. Compounds that showed inhibition percentages higher than 50\% were evaluated again at four concentrations selected according to the $\mathrm{LC}_{50}$ previously obtained for each compound. Infected cells exposed to benznidazol (BNZ) were used as control for anti-trypanosomal activity (positive control) while infected cells incubated in culture medium alone were used as control for infection (negative control). Non-specific absorbance was corrected by subtracting the O.D of the blank. Determinations were done by triplicate in at least two independent experiments [25]. 


\subsubsection{Statistical Analysis}

Cytotoxicity was determined according to the percentages of viability and mortality registered to each compound a concentration, including Benznidazole and culture medium alone. Percentage of viability was calculated by Equation 1, where the O.D of control, corresponds to $100 \%$ of viability.

$$
\% \text { Viability }=(\text { O.D Exposed cells }) /(\text { O.D Control cells }) \times 100
$$

In turn, mortality percentage corresponds to $100-\%$ viability.

Results were expressed as 50 lethal concentrations $\left(\mathrm{LC}_{50}\right)$ that corresponds to the concentration necessary to eliminate $50 \%$ of cells and calculated by Probit analysis [44]. The degree of toxicity was graded according to the $\mathrm{LC}_{50}$ value using the following scale: high cytotoxicity: $\mathrm{LC}_{50}<200 \mu \mathrm{M}$; moderate cytotoxicity: $\mathrm{LC}_{50}>200$ to $<600 \mu \mathrm{M}$ and potentially non-cytotoxicity: $\mathrm{LC}_{50}>600 \mu \mathrm{M}$. Trypanocidal activity was determined according to the percentage of infection obtained for each experimental condition by colorimetry. Parasite inhibition was calculated by equation 1, where the O.D of control corresponds to $100 \%$ of infection.

$$
\% \text { Infection }=(\text { O.D Exposed parasites }) /(\text { O.D Control parasites }) \times 100
$$

In turn, percentage of inhibition of infection corresponds to $100 \%-\%$ of Infection.

Results of antitrypanosomal activity were expressed as $\mathrm{EC}_{50}$ determined by the Probit analysis [44]. antitrypanosomal activity were graded according to the $\mathrm{EC}_{50}$ value using the following scale: High activity: $\mathrm{EC}_{50}<40 \mu \mathrm{M}$, moderate activity: $\mathrm{EC}_{50}>40$ to $<80 \mu \mathrm{M}$; potentially non activity: $\mathrm{EC}_{50}>80$ $\mu \mathrm{M}$. The selectivity index (SI), was calculated by dividing the cytotoxic activity and the trypanocidal activity using the following formula: $\mathrm{SI}=\mathrm{LC}_{50} / \mathrm{EC}_{50}$.

\subsection{Molecular docking against cruzipain structure}

\subsubsection{Structures parameterization}

To verify the potential mechanism of action of the hybrids, the structure of cruzipain, the major papainlike cysteine protease in Trypanosoma cruzi, was obtained from the Protein Data Bank (PDB) [45]. with the id PDB:3I06. The crystal is in complex with a small molecule that acts as inhibitor of the enzymatic activity [46]. For our case, we used the characterized binding site of the complex to test the affinity of the hybrids. The structures of the compounds were 3D-modelled using the server Frog2 [47], which implements a force field to obtain by clustering the 3D structures that are most likely to be 
active, using as input SMILES representations of the molecules. Both, the structures of the selected proteins and the compounds were parameterized using AutoDock Tools [48], as proposed in a previous work [49]. In general, hydrogens were added to polar side chains to facilitate the formation of hydrogen bonds, and the Gasteiger partial charges were calculated. Flexibility was included by considering the torsion angles of the compounds structures.

\subsubsection{Docking and posterior analysis}

The docking search space was delimited with a box containing the binding site identified previously from the crystal. However, the site delimitation was improved with the program Ligsite [50], which is useful to detect pockets using geometrical considerations. Subsequently, the docking simulations were carried out with AutoDock Vina [51]. The exhaustiveness (internal number of repetitions) was 20 for each protein-compound pair. In addition, five replicas per compound were calculated and averaged to obtain the final docking scores in $\mathrm{kcal} / \mathrm{mol}$. The final list was compared with the experimental data using a ranking approach based on the spearman correlation factor. Some of the docked conformations were graphically inspected to check the interactions.

\subsection{Drug-likeness evaluation}

In silico drug-likeness prediction along with further ADMET tools present an array of opportunities which help to accelerate the discovery of new antiparasitic drugs. To find out the drug like properties for all the tested compounds 7a-i and 8a-i, the ADMET physicochemical parameters were determined by QikProp v3.5 module of Schrödinger software. We calculated and analyzed various physicochemical descriptors and pharmaceutical relevant properties such as molecular weight (MW), total polar surface area (TPSA), predicted aqueous solubility (Log S), apparent predicted intestinal permeability (App. Caco-2), prediction of binding to human serum albumin $\left(\log \mathrm{K}_{\mathrm{hsa}}\right)$, number of rotatable bonds, hydrogen bond acceptor site (n-OHNH) and hydrogen bond acceptors (n-ON). Polar surface area was used to calculate the percentage human intestinal absorption (\% HIA) according to the equation \%HIA $=109-[0.345 \mathrm{x}$ PSA] [52]. These important parameters define absorption, permeability, movement and action of drug molecules. 


\section{Acknowledgments}

The authors thank Universidad de Antioquia (grant CODI 6203 and CIDEPRO) for financial support.

\section{Conflict of interest}

The authors declare no conflict of interest.

\section{Supplementary data}

Supplementary data associated with this article can be found, in the online version

\section{References}

[1] World Health Organization. Neglected tropical diseases. Available online: http://www.who.int/neglected diseases/diseases/en/ (accessed on 20 June 2018)

[2] World Health Organization. Chagas disease (American Trypanosomiasis). Available online: http://www.who.int/news-room/fact-sheets/detail/chagas-disease-(american-trypanosomiasis) (accessed on 20 June 2018)

[3] P. Singh, A. Anand, V. Kumar, Recent developments in biological activities of chalcones: a mini review, Eur. J. Med. Chem. 85 (2014) 758-777.

[4] C. Zhuang, W. Zhang, C. Sheng, W. Zhang, C. Xing, Z. Miao. Chalcone: A Privileged Structure in Medicinal Chemistry. Chem. Rev. 117 (2017) 7762-7810.

[5] M.V.P. de Melo, B.A. Abrahim-Vieira, T.F.S. Domingos, J.B. de Jesus, A.C.C. de Sousa, C.R. Rodrigues, A.M.T. Souza. A comprehensive review of chalcone derivatives as antileishmanial agents. Eur. J. Med. Chem. 150 (2018) 920-929.

[6] A.S. Bhambra, K.C. Ruparelia, H.L. Tan, D. Tasdemir, H. Burrel-Saward, V. Yardley, K.J.M. Beresford, R.R.J. Arroo. Synthesis and antitrypanosomal activities of novel pyridylchalcones. Eur. J. Med. Chem. 128 (2017) 213-218.

[7] J.C. Aponte, M. Verastegui, E. Malaga, M. Zimic, M. Quiliano, A.J. Vaisberg, R.H. Gilman, G.B. Hammond, Synthesis, Cytotoxicity, and Anti-Trypanosoma cruzi Activity of New Chalcones. J. Med. Chem. 51 (2008) 6230-6234.

[8] M. Chen, L. Zhai, S.B. Christensen, T.G. Theander, A. Kharazmi, Inhibition of fumarate reductase in Leishmania major and L. donovani by chalcones. Antimicrob. Agents Chemother. 45 (2001) 2023-2029. 
[9] L. Ma, J. Chen, X. Wang, X. Liang, Y. Luo, W. Zhu, T. Wang, M. Peng, S. Li, S. Jie, A. Peng, Y. Wei, L. Chen, Structural modification of honokiol, a biphenyl occurring in magnolia officinalis: the evaluation of honokiol analogues as inhibitors of angiogenesis and for their cytotoxicity and structure-activity relationship, J. Med. Chem. 54 (2011) 6469-6481.

[10] D. Chen, S. Zhang, L. Xie, J. Xie, K. Chen, Y. Kashiwada, B. Zhou, P. Wang, L.M. Cosentino, K. Lee, Anti-aids agents - XXVI. Structure-activity correlations of Gomisin-G-related anti-HIV lignans from Kadsura interior and of related synthetic analogues, Bioorg. Med. Chem. 5 (1997) 1715-1723.

[11] R. Brun, Y. Bühler, U. Sandmeier, R. Kaminsky, C.J. Bacchi, D. Rattendi, S. Lane, S.L. Croft, D. Snowdon, V. Yardley, G. Caravatti, J. Frei, J. Stanek, H. Mett. In Vitro Trypanocidal Activities of New S-Adenosylmethionine Decarboxylase Inhibitors. Antimicrob Agents Chemother 40 (1996) $1442-1447$.

[12] W. Cardona-G, A.F. Yepes, A. Herrera-R, Hybrid Molecules: Promising Compounds for the Development of New Treatments Against Leishmaniasis and Chagas Disease. Curr Med Chem 25 (2018) 3637-3679.

[13] B. Meunier, Hybrid molecules with a dual mode of action: dream or reality? Acc. Chem. Res. 41 (2008) 69-77.

[14] Shaveta, S. Mishra, P. Singh, Hybrid molecules: The privileged scaffolds for various pharmaceuticals. Eur. J. Med. Chem. 124 (2016) 500-536.

[15] E. Otero, E. García, G. Palacios, L.M. Yepes, M. Carda, R. Agut, I.D. Vélez, W.I. Cardona, S.M. Robledo. Triclosan-caffeic acid hybrids: Synthesis, leishmanicidal, trypanocidal and cytotoxic activities. Eur. J. Med. Chem. 141 (2017) 73-83.

[16] S. Vergara, M. Carda, R. Agut, L.M. Yepes, I.D. Vélez, S. Robledo, W. Cardona. Synthesis, antiprotozoal activity and cytotoxicity in U-937 macrophages of triclosanhydrazone hybrids. Med. Chem Res. 26 (2017) 26, 3262-3273.

[17] J.C. Coa, W. Castrillón, W. Cardona, M. Carda, V. Ospina, J.A. Muñoz, I.D. Vélez, S.M. Robledo, Synthesis, leishmanicidal, trypanocidal and cytotoxic activity of quinoline-hydrazone hybrids. Eur. J. Med. Chem., 101 (2015) 746-753.

[18] J.C. Coa, E. García, M. Carda, R. Agut, I.D. Vélez, J.A. Muñoz, L.M. Yepes, S.M. Robledo, W.I. Cardona, Synthesis, leishmanicidal, trypanocidal and cytotoxic activities of quinolinechalcone and quinoline-chromone hybrids. Med. Chem. Res. 26 (2017) 1405-1414. 
[19] E. García, J.C. Coa, E. Otero, M. Carda, I.D. Vélez, S.M. Robledo, W.I. Cardona. Synthesis and antiprotozoal activity of furanchalcone-quinoline, furanchalcone-chromone and furanchalconeimidazole hybrids. Med. Chem. Res. 27 (2018) 497-511.

[20] Z. Qiao, Q. Wang, F. Zhang, Z. Wang, T. Bowling, B. Nare, R.T. Jacobs, J. Zhang, D. Ding, Y. Liu, H. Zhou. Chalcone-Benzoxaborole Hybrid Molecules as Potent Antitrypanosomal Agents. J. Med. Chem. 55 (2012) 3553-3557.

[21] M.A. Ismail, A. Batista-Parra, Y. Miao, W.D. Wilson, T. Wenzler, R. Brunb, D.W. Boykina, Dicationic near-linear biphenyl benzimidazole derivatives as DNA-targeted antiprotozoal agents, Bioorg. Med. Chem. 13 (2005) 6718-6726.

[22] J.T. Pierson, A. Dumètre, S. Hutter, F. Delmas, M. Laget, J.P. Finet, N. Azas, S. Combes, Synthesis and antiprotozoal activity of 4-arylcoumarins. Eur. J. Med. Chem. 45 (2010) 864-869.

[23] V.M. Taylor, D.L. Cedeño, D.L. Muñoz, M.A. Jones, T.D. Lash, A.M. Young, M.H. Constantino, N. Esposito, I.D. Vélez, S.M. Robledo, In vitro and in vivo studies of the utility of dimethyl and diethyl carbaporphyrin ketals in treatment of cutaneous leishmaniasis. Antimicrob Agents Chemother 55 (2011) 4755-4764.

[24] F.S. Buckner, C.L. Verlinde, A.C. La Flamme, W.C. Van Voorhis, Efficient technique for screening drugs for activity against Trypanosoma cruzi using parasites expressing betagalactosidase. Antimicrob Agents Chemother 40 (1996) 2592-2597.

[25] B. Insuasty, J. Ramirez, D. Becerra, C. Echeverry, J. Quiroga, R. Abonia, S.M. Robledo, I.D. Velez, Y. Upegui, J.A. Muñoz, V. Ospina, M. Nogueras, J. Cobo, An efficient synthesis of a new caffeine-based chalcones, pyrazolines and pyrazolo[3-4-b][1-4]diazepines as potential antimalarial, antitrypanosomal and antileishmanial agents. Eur. J. Chem. Med. 93 (2015) 401-413.

[26] W. Cardona, D. Guerra, A. Restrepo, Reactivity of $\delta$-substituted $\alpha, \beta$-unsaturated cyclic lactones with antileishmanial activity. Mo. Simul. 40 (2014) 477-484.

[27] J.C. Mottram, G.H. Coombs, J. Alexander, Cysteine peptidases as virulence factors of Leishmania, Curr Opin Microbiol 7 (2004) 375-381.

[28] J. Aponte, D. Castillo, Y. Estevez, G. Gonzalez, J. Arevalo, G. Hammonda, M. Sauvain, In vitro and in vivo anti-Leishmania activity of polysubstituted synthetic chalcones, Bioorg. Med. Chem. Lett. 20 (2010) 100-103.

[29] M.A. Brenzan, C. Vaturu, B. Dias, T. Ueda, M.C. Young, A. Structure-activity relationship of (-) mammea A/BB derivatives against Leishmania amazonensis. Goncalves, Biomed. Pharmacother. 62 (2008) 651-658.

[30] E. Otero, S.M. Robledo, S. Díaz, M. Carda, D. Muñoz, J. Paños, I.D. Vélez, W. Cardona, 
Synthesis and leishmanicidal activity of cinnamic acid esters: structure-activity relationship, Med. Chem. Res. 23 (2014) 1378-1386.

[31] E. Otero, E. García, G. Palacios, L.M. Yepes, M. Carda, R. Agut, I.D. Vélez, W.I. Cardona, S.M. Robledo, Triclosan-caffeic acid hybrids: Synthesis, leishmanicidal, trypanocidal and cytotoxic activities, Eur. J. M. Chem. 141 (2017) 73-83.

[32] G.L. Patrick, An Introduction to Medicinal Chemistry, fifth ed., Oxford University Press, 2013, pp. 1-14.

[33] M. Sajid, J. H. McKerrow, Cysteine proteases of parasitic organisms. Mol. Biochem. Parasitol. 120 (2002) 1-21.

[34] H.H. Otto, T. Schirmeister, Cysteine Proteases and Their Inhibitors. Chem. Rev. 97 (1997) 133172.

[35] M. Sajid, S.A. Robertson, L.S. Brinen, J.H. McKerrow, Cruzain. In Cysteine Proteases of Pathogenic Organisms, Springer, Boston, MA, (2011). pp. 100-115.

[36] C. Beaulieu, E. Isabel, A. Fortier, F. Massé, C. Mellon, N. Méthot, W.C. Black, Identification of potent and reversible cruzipain inhibitors for the treatment of Chagas disease. Bioorg. Med Chem. Let. 20 (2010) 7444-7449.

[37] R. Li, X. Chen, B. Gong, P.M. Selzer, Z. Li, E. Davidson, G. Kurzban, R.E. Miller, E.O. Nuzum, J.H. McKerrow, R.J. Fletterick, S.A. Gillmor, C.S. Craik, I.D. Kuntz, F.E. Cohen, G.L. Kenyon, Structure-based design of parasitic protease inhibitors. Bioorg. Med. Chem. 4 (1996) 1421-1427.

[38] L. Troeberg, X. Chen, T.M. Flaherty, R.E. Morty, M. Cheng, H. Hua, C. Springer, J.H. McKerrow, G.L. Kenyon, J.D. Lonsdale-Eccles, T.H.T. Coetzer, F.E. Cohen, Chalcone, Acyl Hydrazide, and Related Amides Kill Cultured Trypanosoma brucei brucei. Molec. Med. 6 (2000) 660-669.

[39] D.M. Borchhardt, A. Mascarello, L.D. Chiaradia, R.J. Nunes, G. Oliva, R.A. Yunes, A.D. Andricopulo, Biochemical evaluation of a series of synthetic chalcone and hydrazide derivatives as novel inhibitors of cruzain from Trypanosoma cruzi. J. Braz. Chem. Soc. 21 (2010) 142-150.

[40] D. Gomes-Vital, M. Arribas, G.H. Goulart-Trossini, Molecular modeling and docking application to evaluate cruzain inhibitory activity by chalcones and hydrazides. Lett. Drug Des. Discov. 11 (2014) 249-255.

[41] C.A. Lipinski, F. Lombardo, B.W. Dominy, P.J. Feeney, Experimental and computational approaches to estimate solubility and permeability in drug discovery and development settings. 
Adv. Drug Deliv Rev. 23(1997) 3-25.

[42] P. Ertl, B. Rohde, P. Selzer, Fast calculation of molecular polar surface area as a sum of fragment based contributions and its application to the prediction of drug transport properties. J. Med. Chem. 42 (2000) 3714-3717.

[43] D.F. Veber, S.R. Johnson, H.-Y. Cheng, B.R. Smith, K.W. Ward, K.D. Kopple, Molecular properties that influence the oral bioavailability of drug candidates. J.Med.Chem. 45 (2002) 26152623.

[44] Finney JD (1978) Probit Analysis: Statistical Treatment of the Sigmoid Response Curve, $3^{\text {rd }}$ ed.; Cambridge University Press: Cambridge, UK, p. 550.

[45] H.M. Berman, T. Battistuz, T.N. Bhat, W.F. Bluhm, P.E. Bourne, K. Burkhardt, P. Fagan, The protein data bank. Acta Crystallogr. D Biol. Crystallogr. 58 (2002) 899-907.

[46] B.T. Mott, R.S. Ferreira, A. Simeonov, A. Jadhav, K.K.H. Ang, W. Leister, J. H. McKerrow, Identification and optimization of inhibitors of trypanosomal cysteine proteases: cruzain, rhodesain, and TbCatB. J. Med. Chem. 53 (2009) 52-60.

[47] M.A. Miteva, F. Guyon, P. Tuffiery, Frog2: Efficient 3D conformation ensemble generator for small compounds. Nucleic acids res. 38 (suppl_2) (2010) W622-W627.

[48] G.M. Morris, R. Huey, W. Lindstrom, M.F. Sanner, R.K. Belew, D.S. Goodsell, A.J. Olson, AutoDock4 and AutoDockTools4: Automated docking with selective receptor flexibility. J. Comput. Chem. 30 (2009) 2785-2791.

[49] R. Ochoa, S.J. Watowich, A. Flórez, C.V. Mesa, S.M. Robledo, C. Muskus, Drug search for leishmaniasis: a virtual screening approach by grid computing. J. Comput. Aided Mol. Des. 30 (2016) 541-552.

[50] Huang, B., \& Schroeder, M. (2006). LIGSITE csc: predicting ligand binding sites using the Connolly surface and degree of conservation. BMC structural biology, 6(1), 19.

[51] O. Trott, A.J. Olson, AutoDock Vina: improving the speed and accuracy of docking with a new scoring function, efficient optimization, and multithreading. J. Comput. Chem. 31 (2010 455-461.

[52] Y. Zhao, M.H. Abraham, J. Lee, A. Hersey, N.C. Luscombe, G. Beck, B. Sherborne, I. Cooper, Rate-limited steps of human oral absorption and QSAR studies. Pharm. Res. 19 (2002) 1446-1457. 